Please do not remove this page

RMIT

UNIVERSITY

\title{
Colloquium: An algebraic model of localized surface plasmons and their interactions
}

Davis, Timothy; Gomez, Daniel

https://researchrepository.rmit.edu.au/esploro/outputs/9921860355501341/filesAndLinks?institution=61RMIT_INST\&index=null

Davis, T., \& Gomez, D. (2017). Colloquium: An algebraic model of localized surface plasmons and their interactions. Reviews of Modern Physics, 89(1), 1-20. https://doi.org/10.1103/RevModPhys.89.011003 Document Version: Accepted Manuscript

Published Version: https://doi.org/10.1103/RevModPhys.89.011003

Repository homepage: https://researchrepository.rmit.edu.au

(C) 2017 American Physical Society

Downloaded On 2023/04/26 21:06:40 +1000

Please do not remove this page 


\title{
Colloquium: An algebraic model of localized surface plasmons and their interactions
}

\author{
T. J. Davis \\ School of Physics, University of Melbourne, Parkville Victoria Australia and \\ $4^{\text {th }}$ Physics Institute and Research Center SCOPE, University of Stuttgart, Pfaffenwaldring 57, 70550 Stuttgart, \\ Germany \\ D. E. Gómez
}

CSIRO, Manufacturing, Private Bag 33, Clayton, VIC, 3168, Australia and

School of Physics, University of Melbourne, Parkville Victoria Australia

\begin{abstract}
Although localized surface plasmons in metal nanoparticles can be modelled by Maxwells equations, the difficulty in solving them forces many researchers to use numerical methods. Such methods give accurate results but rarely provide much insight into the complex behaviors of the surface plasmons, nor do they provide a means to choose a configuration of metal nanoparticles to achieve a desired optical response. This Colloquium presents a simple algebraic approach for modelling localized surface plasmons, their excitation by light and their interactions with one another, which yields useful insights into plasmon behaviour and enables the design of complex plasmonic devices. The approach is similar to the matrix methods developed for quantum mechanics and relies on a description of the surface plasmons in terms of a set of eigenmodes. As in quantum mechanics, the functional form of these modes are not usually required and the entire problem is reduced to a simple algebra involving the plasmon amplitudes, resonance terms and their mutual coupling. In this Colloquium, the algebraic method is derived from an electrostatic formalism and then used to demonstrate a variety of optical effects associated with localized surface plasmons, such as plasmon hybridization, induced transparency, Fano resonances, optical phase detection and all-optical modulation, among others.
\end{abstract}

\section{Contents}

\section{Introduction}

11. A theory of localized surface plasmon resonances

A. The "electrostatic" eigenvalue problem

B. LSP excitation amplitudes

C. Radiation

D. Radiation damping

E. Properties of isolated metal nanoparticles

F. Coupled LSPS

III. Analysing interactions between coupled LSPS

A. Two particle coupling, dark modes and Fano resomances

1. Plasmon hybridization

2. Bright and dark modes

3. Fano resonances

B. Coupling in three nanorod structures

1. Plasmon-induced transparency

2. Plasmonic Wheatstone bridge

3. All-optical modulation

4. Radially-symmetric structures and dark modes

C. Coupling in 3D structures

IV. Concluding remarks

References

S1. The eigenmodes and eigenvalues of Eqn. (4) for a sphere

S2. Drude model and the electostatic approximation

\section{INTRODUCTION}

2

5

6

7

8

8

9

9

10

10

11

11

12

13

14

15

15

18

19
Localized surface plasmons (LSPs) are oscillations of the conduction electrons excited by light on the surfaces of metal nano-particles (Barnes et al., 200:3; Kreibig and Vollmer, ए99.5; Maier, 20107; Odom and Schat7, 2011; Ozbay, 2006; Raether, 1977, 1988; Zayats et al., 2005). There has been great interest in LSPs among scientists and engineers, due in part to their properties such as confining light to nanoscale volumes (Bozhevolnyi et al., 20106), preserving phase coherence (Fakonas et al., 2014), enabling light energy and spectral content to be manipulated (Kim et al., 11999; Li et al., 2014), preserving quantum coherence (Chang et al., 2006). These properties depend on the geometry and composition of the metal nanoparticles, their dielectric environment and also on their mutual interactions when arranged in close proximity to each other. Moreover, using coupled LSPs and their interference to manipulate optical resonances creates unusual optical effects, such plasmon "induced" transparency, suppressed light scattering (dark modes), Fano resonances and all-optical modulation, among others. The problem is to understand how the complex interplay between these parameters modifies the optical properties of the nanoparticles and how one can design a configuration of coupled metal nano-particles to achieve a desired optical effect. There are many methods that can be used to model the interaction of light with LSPs, ranging from simple coupled oscillator models (Lill et al, 2009; Lovera et al., 2013; Taubert et al., 2012; Zhang et al., 2008$)$ to full analytical and numerical solutions to 
Maxwell's equations (Lalanne and Jurek, 11998; Lalanne and Morris, 1096; Moharam et al., 1995; Weiss et al., 200.9). However, whatever method is chosen, it is important for understanding and designing optical devices that it yields some physical insight into the nature of the interactions and their effects on light fields.

The aim of this colloquium is twofold. Firstly we present a physical description of coupled LSPs, based on an expansion in terms of eigenmodes (or fundamental resonances), that enables the rational design of complex plasmonic structures without the need for time and resource consuming numerical calculations. Secondly, we use this algebraic model to describe some of the important experimental observations that arise from localized surface plasmon resonance phenomena.

\section{A THEORY OF LOCALIZED SURFACE PLASMON RESONANCES}

Localized surface plasmons (LSPs) excited by light on metal nanoparticle surfaces are observed experimentally by strong scattering at a particular frequency - the resonance frequency of the LSP. Such resonances have been known for a long time. In spherical metal nanoparti-

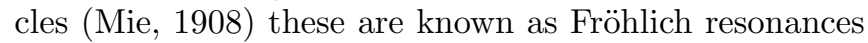
(Bohren and Huftman, 198.3) and are the fundamental electric dipole resonances of spheres.

So how do the resonances come about and how can we model them mathematically? What happens when two or more metal particles are close to one another and their resonating electric fields overlap?

The surface plasmons are well-described by classical electromagnetism embodied in Maxwell's equations. One of the simplest cases is a semi-infinite metal-dielectric interface (Raether, 1.977, 1988). If we consider an electromagnetic wave at the interface and seek a solution involving outwardly propagating waves only, we find a wave travelling along the interface with a wavenumber $\alpha=k\left(\epsilon_{m} \epsilon_{b} /\left(\epsilon_{m}+\epsilon_{b}\right)\right)^{1 / 2}$, where $\epsilon_{m}$ and $\epsilon_{b}$ are the respective electric permittivities of the metal and the dielectric, and $k=\omega / c$ is the free-space wavenumber. At visible and infrared frequencies, the electric permittivity of a metal is negative $\epsilon_{m}<0$ and large $\left|\epsilon_{m}\right| \gg \epsilon_{b}$ which means that $\alpha$ is complex and larger than the free-space wavevector $(|\alpha|>k)$. Such a wave cannot radiate into free space and is therefore trapped at the metal surface. This wave is a surface plasmon polariton - a wave of surface charge propagating over the metal surface (Raether, 1977) coupled with its electromagnetic field in the adjacent dielectric medium. The electric field is strong at the surface but decays exponentially with distance into the metal and into the adjacent dielectric medium. This decaying field is known as an evanescent wave.

The surface plasmon wave reflects from discontinuities in the film, such as a dielectric ridge or a defect in the metal surface (Zayats et al., 2005). If the film contains multiple boundaries, a surface plasmon stand- ing wave can arise at certain frequencies where the multiple reflections overlap in phase (Pacifici et al., 20107; Schouten et al. 2000.5$)$. If we now think of surface plasmon waves propagating over the surface of a metal particle, the waves travel around the surface, overlap and interfere. When the path length is an integer number of surface plasmon wavelengths, constructive interference occurs and a standing wave is formed. This condition occurs only at specific frequencies of the incident light: these standing waves are the localized surface plasmon resonances.

Although Maxwell's equations can describe the LSPs, the solutions are very difficult to obtain analytically and are generally not available for arbitrarily shaped metal nanoparticles. One of the problems relates to the interaction between the time-varying electric and magnetic fields. The surface plasmons are excited by the incident electric fields that oscillate in time producing oscillating magnetic fields. The oscillating magnetic fields react back on the charges, inducing displacement currents (Faraday's law) opposing the electron motion and modifying the resonant frequencies. In effect, the electromagnetic waves emitted from one region on the nanoparticle propagate through space, suffer a phase delay and then interact with another nanoparticle region.

The problem is greatly simplified when the metal particles are very much smaller than the wavelength of light. Under this condition the magnetic fields from the oscillating surface charges are small. Magnetic effects scale with the wavenumber $k$ and the displacement electric fields reacting back on the surface charges scale as $k^{2}$. For very small metal particles we find that the electric effects decouple from the magnetic effects and the problem takes a mathematical form identical to that in electrostatics. Another way to see this is to write down the electric field radiated from an oscillating dipole $\mathbf{p}$ in direction $\hat{r}$ (Jackธणा, [1.975)

$$
\begin{aligned}
\mathbf{E} & =\frac{e^{i k r}}{4 \pi \epsilon_{0} r^{3}}\left[(k r)^{2}(\hat{r} \times \mathbf{p}) \times \hat{r}-i k r(3 \hat{r}(\hat{r} \cdot \mathbf{p})-\mathbf{p})\right] \\
& +\frac{e^{i k r}}{4 \pi \epsilon_{0} r^{3}}(3 \hat{r}(\hat{r} \cdot \mathbf{p})-\mathbf{p}) .
\end{aligned}
$$

For distances such that $k r \ll 1$ only the last term in Eq. (四) remains. This is the radiation near-field which has exactly the same form as the electrostatic result: that is "the fields in the near zone are dominantly electric in nature" (Jackson, ए975).

\section{A. The "electrostatic" eigenvalue problem}

The electrostatic theory describing the LSPs in nanoparticles with dimensions much smaller than the wavelength of light has its origins in potential theory, which describes systems of charges and dipoles and the potentials they produce (Kellog, 1929). The method was used to model the energy loss of electrons to surface 
plasmons when passing by a metal particle (Ouyang and Isaacson, 1989). As we show below, the solution is found from an eigenvalue problem describing the self-consistent charge distributions or standing waves on the surface of a metal particle. The eigenvalues describe the resonances and can be considered a form of spectral theory. Such theories were investigated by Bergman (Bergman,

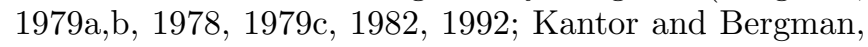
1982) who used them to model the electromagnetic properties of mixtures of metal particles and dielectrics.

The origin of the surface plasmon mode is a selfsustained surface charge oscillation: an electric charge on the surface of the metal produces an electric field that interacts with other charges on the metal surface (Fig. $\square(a)-(b)$ ). The electric field from a charge at some point $\mathbf{r}^{\prime}$ extends through the background medium of electric permittivity $\epsilon_{b}$ surrounding the metal and then penetrates the metal surface at another point $\mathbf{r}$ inducing a surface charge $\sigma$. The amount of surface charge induced at $\mathbf{r}$ is found from the discontinuity of the electric field through the metal surface $\left(\mathbf{E}_{b}-\mathbf{E}_{m}\right) \cdot \hat{n}=\sigma / \epsilon_{0}$ that depends on the direction of the surface normal $\hat{n}$. It is straightforward to show that a surface charge distribution $\sigma$ produces an electric field $\mathbf{E}_{b} \cdot \hat{n}=\sigma / 2 \epsilon_{0}$ on the space outside it and $\mathbf{E}_{m} \cdot \hat{n}=-\sigma / 2 \epsilon_{0}$ on the inside of the surface.

However, all charges on the metal surface contribute to this surface charge, and likewise, this charge produces an electric field that also interacts with all points on the metal surface. The problem is to find the distribution of surface charges in a self-consistent manner. We first write Coulomb's law for the electric field from the electric charge $q\left(\mathbf{r}^{\prime}\right)=\sigma\left(\mathbf{r}^{\prime}\right) d S^{\prime}$ arising from each patch of surface with area $d S^{\prime}$

$$
\mathbf{E}(\mathbf{r})=\frac{1}{4 \pi \epsilon_{0}} \oint \sigma\left(\mathbf{r}^{\prime}\right) \frac{\left(\mathbf{r}-\mathbf{r}^{\prime}\right)}{\left|\mathbf{r}-\mathbf{r}^{\prime}\right|^{3}} d S^{\prime}
$$

The electric field at position $\mathbf{r}$ on the surface is due to the field from all the surface charges plus the charge at position $\mathbf{r}$, given by the boundary condition on the normal component (Kellog, [929). Thus the normal components of the fields in the metal and the dielectric are given by

$$
\mathbf{E}_{m, b}(\mathbf{r}) \cdot \hat{n}=\mp \frac{\sigma(\mathbf{r})}{2 \epsilon_{0}}+\frac{1}{4 \pi \epsilon_{0}} \oint \sigma\left(\mathbf{r}^{\prime}\right) \frac{\hat{n} \cdot\left(\mathbf{r}-\mathbf{r}^{\prime}\right)}{\left|\mathbf{r}-\mathbf{r}^{\prime}\right|^{3}} d S^{\prime}
$$

Since the normal component of the electric displacement vector $\mathbf{D}=\epsilon \mathbf{E}$ is continuous across a boundary, we can relate the fields either side of the metal surface $\epsilon_{m} \mathbf{E}_{m} \cdot \hat{n}=$ $\epsilon_{b} \mathbf{E}_{b} \cdot \hat{n}$. Placing the electric fields from Eq. (Bi) into this boundary condition leads to the condition required for self-consistency(Mayergoyz et al., 2005; Ouyang and Isaacson, ए989):

$$
\sigma(\mathbf{r})=\frac{\gamma}{2 \pi} \oint \sigma\left(\mathbf{r}^{\prime}\right) \frac{\hat{n} \cdot\left(\mathbf{r}-\mathbf{r}^{\prime}\right)}{\left|\mathbf{r}-\mathbf{r}^{\prime}\right|^{3}} d S^{\prime}
$$

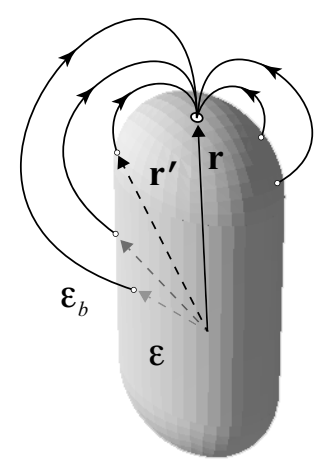

a)

FIG. 1 The surface charges and surface dipoles that give rise to localized surface plasmon resonances. a) the charge at each point $\mathbf{r}$ induced by the electric fields from all the other charges at $\mathbf{r}^{\prime}$ distributed over the surface of a nanoparticle; b) The LSP can be described by a distribution of surface charges $\sigma$; c) or by a double-layer of charge that is modelled by a distribution of surface dipoles $\tau$.

where the eigenvalue

$$
\gamma=\frac{\epsilon_{m}(\omega)-\epsilon_{b}}{\epsilon_{m}(\omega)+\epsilon_{b}}
$$

depends on the electric permittivity of the metal $\epsilon_{m}(\omega)$ and that of the surrounding, or background, medium $\epsilon_{b}$. The equation for the induced surface charge has the form of an integral eigenvalue problem $\left(L-\lambda_{L}\right) \sigma=0$ where $L$ is the integral operator, $\lambda_{L}=(2 \pi / \gamma)$ is its eigenvalue and $\sigma$ is the eigenfunction. For simplicity we shall refer to $\gamma$ as the eigenvalue of the solution, although strictly the eigenvalue problem yields $\lambda_{L}$.

Like all eigenvalue problems we obtain a series of eigenvalues and an associated set of eigenfunctions. We label these to distinguish them from one another. We associate the $k$-th eigenfunction $\sigma_{n}^{k}(\mathbf{r})$ with the $k$-th eigenvalue $\gamma_{n}^{k}$ of particle $n$. Examples of the eigenfunctions of a "flattened cylinder" are shown in Fig. $\square$ which were found by numerically solving Eq. (四) using the method of Mayergoyz (Mayergoyz et al., 200.5) (see also the freelyavailable Boundary Element Method Matlab toolbox by Hohenester and Trugler (2012) in its electrostatic limit). Analytical solutions are also possible for special nanoparticle geometries such as a sphere, for which we show in section $\boldsymbol{\Sigma}]$ the eigenmodes and eigenvalues obtained by solving Eq. (四).

The eigenfunctions of Eq. (⿴囗十) are the standing waves on the surface of the particle, not unlike the standing waves one might find on the surface of a vibrating membrane. These surface charge distributions have a complicated multi-polar nature, meaning the distributions can be expanded in terms of dipoles, quadrupoles, hexapoles and so on (Wei et al, 2010). The fundamental resonance, being the first one shown in Fig. $\mathbf{Z}$, has a strong dipole 
characteristic. This is common to most nanostructures and we will exploit this property below. Each standing wave is associated with an eigenvalue which is a real valued number greater than one. Note that the surface charges oscillate in time but a common factor $e^{-i \omega t}$ cancels out on both sides of equation (田).

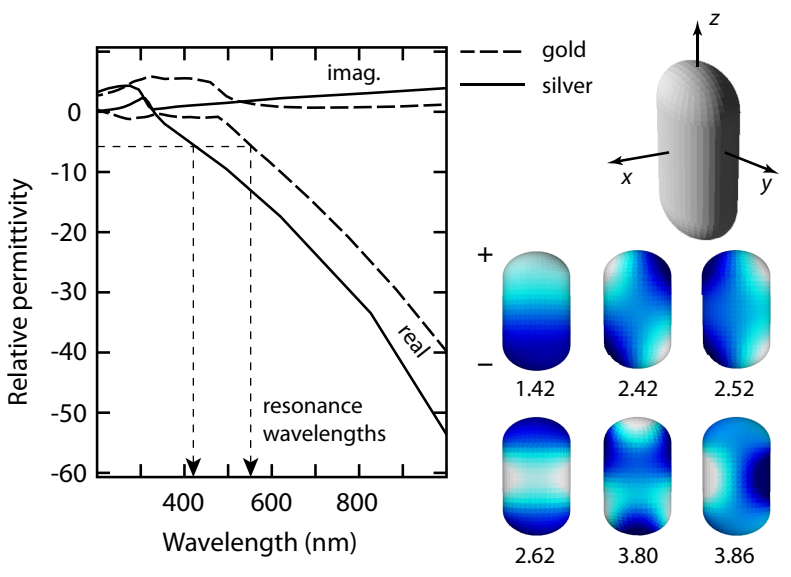

FIG. 2 The first six eigenmodes of an example particle, which is a flattened cylinder with rounded ends. The colour represents the surface dipole distribution and the numbers are the eigenvalues. Also shown are the real and imaginary parts of the relative electric permittivities of gold and silver. For the fundamental dipole mode $\gamma_{1}^{1}=1.42$ placed in air $\epsilon_{b}=1$ the resonance occurs where the metal permittivity $\epsilon=\epsilon_{b}(1+\gamma) /(1-\gamma)=-5.76$ at wavelengths $554 \mathrm{~nm}$ for gold and $426 \mathrm{~nm}$ for silver as shown.

The problem as outlined in Eq. (田) is scale independent. Changing the dimensions of length such as $r \rightarrow \beta r$ leaves the equation unchanged since all powers of $\beta$ cancel. Thus we find that the eigenfunctions only depend on the geometry of the nanoparticle, and in particular on its aspect ratio, but not on its actual size. This is a consequence of the "electrostatic" or near-field approximation used in this theory. Another important feature of Eq. (四) is that it places no restrictions on the continuity of the surface - that is the surface may consist of several pieces. In this respect the eigenvalue problem can be applied to two or more particles of arbitrary shape and it will give the LSP resonances of the combination (Gómez et al. 2(10). We shall use this fact below to derive an expression describing the interaction between LSPs on multiple particles.

Once the eigenvalue $\gamma$ has been found and a background medium specified, then equation (国) requires the electric permittivity $\epsilon$ to satisfy: $\epsilon(\omega)=\epsilon_{b}(1+\gamma) /(1-\gamma)$. With usual dielectrics $\epsilon_{b}>0$, and given that $\gamma \geq 1$, then equation (5) is satisfied only for materials with $\epsilon(\omega)<0$. This is mainly the case for metals, semi-metals and some semi-conductors. Since the electrostatic problem (团) is real, it requires the permittivities to be real. We shall reconsider this point later in the context of LSP excitation amplitudes. It is important to note that the electric permittivity of a metal is negative and changes with fre- quency. The frequency $\omega_{R}$ at which the metal permittivity satisfies the eigenvalue condition is the frequency at which the surface charges oscillate: this is, in fact, the LSP resonance. Since these resonances arise as a consequence of an "electrostatic" or near-field condition, these are sometimes referred to as "electrostatic" resonances. Figure $\square$ shows the real and imaginary parts of the relative electric permittivities of gold and silver (Weaver and Frederikse, 2(106) and the wavelengths at which the fundamental dipole resonance of the example nanoparticle will resonate in air, based on the real part of the permittivity.

Surface charges are one way to describe an electrostatic problem but we can also use surface dipole distributions. In this model the boundary condition arise from a change in the electric potential across the surface, which is then related to the surface dipole moment $\phi_{b}-\phi_{m}=\tau / \epsilon_{0}$ ("Tackson, 1975). In effect, an electric charge on the surface has a companion opposite charge just below the surface, creating a surface dipole moment $\tau \hat{n}$, also known as a double layer (Kellog, 1929), aligned parallel to the surface normal $\hat{n}$ (Fig. 四(c)). The surface dipole moment also obeys an eigenvalue problem

$$
\tau(\mathbf{r})=\frac{\gamma}{2 \pi} \oint \tau\left(\mathbf{r}^{\prime}\right) \frac{\hat{n}^{\prime} \cdot\left(\mathbf{r}^{\prime}-\mathbf{r}\right)}{\left|\mathbf{r}^{\prime}-\mathbf{r}\right|^{3}} d S^{\prime}
$$

This is the "dual" problem associated with the surface charge eigenvalue equation.

Both equations (田) and (6) are equivalent and lead to

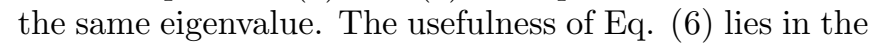
property that the surface charge and surface dipole eigenfunctions form a biorthogonal set: $\oint \tau_{m}^{j}(\mathbf{r}) \sigma_{n}^{k}(\mathbf{r}) d S=$ $\delta_{m n} \delta^{j k}$. The integral is zero unless the eigenfunctions are associated with the same particle and the same eigenvalue. We use superscripts on the delta function to remind us these refer to the eigenmodes (not to be confused with the notation used in relativity to refer to covariant and contravariant vectors). This is similar to the orthonormal set of functions $\psi_{n}$ used in quantum mechanics as derived from the Schrödinger equation. The difference is that the quantum wavefunction is Hermitian so that its adjoint is equal to the complex conjugate $\psi_{n}^{\dagger}=\psi_{n}^{*}$ leading to $(1 / V) \int \psi_{n}^{*}(\mathbf{r}) \psi_{m}(\mathbf{r}) d V=\delta_{n m}$. Here the electrostatic eigenfunctions are real and do not have this property, which requires the use of an auxiliary equation resulting in a bi-orthogonal pair $\sigma_{n}^{k}$ and $\tau_{m}^{j}$. In the language of linear algebra, these two functions form a left and a right eigenvector pair.

\section{B. LSP excitation amplitudes}

In the previous section we described the LSPs in terms of the natural resonant modes of a nanoparticle that has a negative electric permittivity. The natural resonances are described mathematically by surface charge eigenfunctions, surface dipole eigenfunctions and their eigenvalues. The question arises as to which of these modes, if 
any, are exited when an electromagnetic wave is incident on the particle and how strong are the excitations?

This problem was addressed in the context of nanoparticle excitation by pulsed laser sources (Mayergoyz et al. , 2007). The electric field $\mathbf{E}_{0}(\mathbf{r}) \exp (-i \omega t)$ of the incident light excites an oscillating surface charge $\sigma(\mathbf{r}, \omega) \exp (-i \omega t)$ that varies with position over the nanoparticle surface. This surface charge can be represented by a linear combination of the eigenmodes of the particle: $\sigma(\mathbf{r}, \omega)=\sum_{k} a_{n}^{k}(\omega) \sigma_{n}^{k}(\mathbf{r})$. The expansion coefficient $a_{n}^{k}(\omega)$ represents the strength of the excitation of the $k$-th LSP mode: the excitation amplitude. By applying the boundary condition that the normal components of the electric displacement across the metal surface are continuous, then $\epsilon_{m}(\omega)\left(\mathbf{E}_{0}+\mathbf{E}_{m}\right) \cdot \hat{n}=\epsilon_{b}\left(\mathbf{E}_{0}+\mathbf{E}_{b}\right) \cdot \hat{n}$. Across the boundary at a position $\mathbf{r}$, the normal component of the electric field has a discontinuity given in Eq. (Bil). Substituting for $\mathbf{E}_{m}$ and $\mathbf{E}_{b}$, expanding the surface charge in terms of eigenfunctions, multiplying by $\tau_{m}^{j}(\mathbf{r})$, integrating over the surface and using the biorthogonality condition, leads to an equation for the excitation amplitude

$$
\begin{aligned}
a_{m}^{j}(\omega) & =f_{m}^{j}(\omega) \oint \tau_{m}^{j}\left(\mathbf{r}^{\prime}\right) \hat{n}^{\prime} \cdot \mathbf{E}_{0}\left(\mathbf{r}^{\prime}\right) d S^{\prime} \\
& \approx f_{m}^{j}(\omega) \mathbf{p}_{m}^{j} \cdot \mathbf{E}_{0}
\end{aligned}
$$

where

$$
f_{m}^{j}(\omega)=\frac{2 \gamma_{m}^{j} \epsilon_{b}\left(\epsilon_{m}(\omega)-\epsilon_{b}\right)}{\epsilon_{b}\left(\gamma_{m}^{j}+1\right)+\epsilon_{m}(\omega)\left(\gamma_{m}^{j}-1\right)}
$$

We have introduced a factor $f_{m}^{j}(\omega)$, which is the polarizability per unit volume. As we show below this factor plays a key role in determining the LSP resonance and includes information on the amplitudes and the phases.

At the subwavelength scale where the electrostatic limit applies, the incident electric field is approximately constant over the nanoparticle surface. Under this condition $\mathbf{E}_{0}$ can be taken out of the integral leading to $a_{m}^{j}(\omega) \approx f_{m}^{j}(\omega) \mathbf{p}_{m}^{j} \cdot \mathbf{E}_{0}$ as given in Eq. (घ) where $\mathbf{p}_{m}^{j}=\oint \tau_{m}^{j}\left(\mathbf{r}^{\prime}\right) \hat{n}^{\prime} d S^{\prime}$ represents the average dipole moment associated with the eigenfunction $\tau_{m}^{k}$. This dipole moment is proportional to the actual dipole moment of the LSP mode $k$ in the nanoparticle, differing only by a scale factor. Indeed, the true dipole moment is just $\mathbf{p}_{m}(\omega)=\sum_{k} a_{m}^{k}(\omega) \mathbf{p}_{m}^{k}$. In what follows we shall refer to $\mathbf{p}_{m}^{k}$ as the eigenfunction dipole moment to distinguish it from the true dipole moment $\mathbf{p}_{m}$. Note that we can also write the eigenfunction dipole moment in terms of the surface charge $\mathbf{p}_{m}^{j}=\oint \sigma_{m}^{j}\left(\mathbf{r}^{\prime}\right) \mathbf{r}^{\prime} d S^{\prime}$ which is identical to the eigenfunction dipole moment derived from $\tau_{n}^{k}$ if the eigenfunctions are properly normalised (Davis et all, 2010b).

The excitation amplitude $a_{m}^{j}(\omega)$ represents the strength of the LSP mode $j$ excited on nanoparticle $m$ by the incident light field at frequency $\omega$. The frequency dependence is contained within the factor $f_{m}^{j}(\omega)$. This factor becomes very large when the real part of the denominator is zero, $\Re\left[\epsilon_{b}\left(\gamma_{m}^{j}+1\right)+\epsilon(\omega)\left(\gamma_{m}^{j}-1\right)\right]=0$, which is the same relationship we found previously linking the eigenvalue to the electric permittivities of the nanoparticle and the surrounding medium (Eq. (回)). The excitation amplitude is a complex quantity because it contains, through $f_{m}^{j}$, information about the phase of the LSP oscillation relative to the phase of the driving field $\mathbf{E}_{0}$. For frequencies away from resonance we expect $f_{m}^{j}$ to become small, reducing the strength of the LSP.

An approximate expression for the frequency dependence of $f_{m}^{j}(\omega)$ can be found using a Drude model for the metal permittivity (Ashcroft and Mermin, 1976; Kittell, 19.56). In this model the electric permittivity is given by a form $\epsilon_{m}(\omega) \approx 1-\omega_{P}^{2} / \omega(\omega+i \Gamma)$ where $\omega_{P}$ is the bulk plasma frequency of the metal and $\Gamma$ is a loss term. Using this expression in Eq. (8), expanding the result about the resonance frequency $\omega_{R}$ and assuming the loss $\Gamma$ is small, then the frequency-dependent factor can be approximated by

$$
\begin{aligned}
f_{m}^{j}(\omega) & \approx-\left(\frac{2 \gamma_{m}^{j} \epsilon_{b}^{2} \omega_{m}^{j}{ }^{2}}{\left(\gamma_{m}^{j}-1\right)^{2} \omega_{P}^{2}}\right) \frac{1}{\left(\omega-\omega_{m}^{j}+i \Gamma_{m}^{j} / 2\right)} \\
& =\frac{-A_{m}^{j}}{\omega-\omega_{m}^{j}+i \Gamma_{m}^{j} / 2},
\end{aligned}
$$

where $\omega_{m}^{j}$ is the resonance frequency of mode $j$ of particle $k, \Gamma_{m}^{j}$ is the full-width at half maximum (FWHM) of the resonance, and $A_{m}^{j}$ is a positive scale factor that depends on the resonant mode and the permittivity of the background medium (a detailed derivation of this approximate form is shown in section $\mathbf{5 2}$ ).

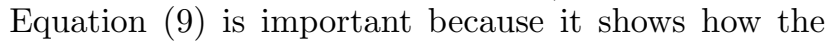
LSP resonance changes with frequency and it enables us to model in a simple way the phase variations of the resonance relative to the incident electric field. This equation is approximate and is only valid close to the LSP resonance. This equation, along with Eq. (U), are the key expressions that we use to model LSP resonances in single metal nanoparticles. As we show below, the properties of LSPs in ensembles of metal nanoparticles can be obtained from the mathematical form of Eqs. (ש) and (四) without needing to know the actual values of the resonance frequency, the FWHM, the scale factor or even the eigenvalues or eigenfunctions! This is analogous to problems in quantum mechanics (based on Dirac's braket notation, $\langle\psi|$ and $|\psi\rangle)$ in which many properties of quantum systems can be determined using the linear algebra of the operators, the wavefunctions and their orthogonality properties without requiring actual details of the spatial dependence or their functional form (CohenTannoudji et al., ए.977).

\section{Radiation}

In this theory we have neglected radiation, since all the electric fields are evanescent. Light radiated from 
these structures must be added as a separate effect. Since the dipole resonance dominates over higher-order multipoles, we can write the radiated power in terms of the LSP dipole moments. For a single nanostructure the total induced surface charge is $\sigma_{n}(\mathbf{r})=\sum_{j} a_{n}^{j}(\omega) \sigma_{n}^{j}(\mathbf{r})$ and the dipole moment $\mathbf{p}_{n}=\oint \sigma_{n}(\mathbf{r}) \mathbf{r} d S$ is then $\mathbf{p}_{n}=$ $\sum_{j} a_{n}^{j}(\omega) \mathbf{p}_{n}^{j}$ where $\mathbf{p}_{n}^{j}$ is the eigenvector dipole moment of LSP mode $j$ of particle $n$. The time-averaged power per solid angle radiated in the far field direction $\hat{n}$ can be written in the form (1ackson, 1975)

$$
\frac{d P}{d \Omega}=\frac{c k^{4}}{32 \pi^{2} \epsilon_{b}}(\hat{n} \times \mathbf{p}) \cdot\left(\hat{n} \times \mathbf{p}^{*}\right),
$$

where $c$ is the speed of light, $\epsilon_{b}$ is the relative permittivity of the dielectric occupying the space and $\mathbf{p}^{*}$ is the complex conjugate of the dipole moment.

For a particle with a single LSP mode it is clear the radiated power is proportional to $\left|a_{n}\right|^{2}$. The scattering cross-section of a particle $C_{\mathrm{sca}}=\left(k^{4} / 6 \pi\right)\left|\mathbf{p} / E_{0}\right|^{2}$ is also proportional to the modulus square of the dipole moment (Bohren and Huftman, 1.983) leading to a similar dependence on $\left|a_{n}\right|^{2}$ of the excitation amplitude. This provides a convenient way to estimate the relative strength of the light re-radiated by the LSPs.

\section{Radiation damping}

An oscillating surface charge density (with an associated dipole moment $\mathbf{p}$ ) will radiate electromagnetic energy. This radiation is one mechanism by which the resonance is damped, increasing the spectral linewidth and decreasing the quality factor. The radiated electromagnetic field can be equated to a "self-field" (Meystre and Sargent, [1998) $\mathbf{E}_{\text {rad }}=i k^{3} /\left(6 \pi \epsilon_{b}\right) \mathbf{p}$ that perturbs the oscillation $(k=\omega / c)$. This field can be included in the expression for the excitation amplitude of Eq. ([U) (Davis et al. 2009b)

$$
\begin{aligned}
a_{m}^{j}(\omega) & =f_{m}^{j}(\omega) \mathbf{p}_{m}^{j} \cdot\left(\mathbf{E}_{0}+i \frac{k^{3}}{6 \pi \epsilon_{b}} \mathbf{p}\right) \\
& =f_{m}^{j}(\omega) \mathbf{p}_{m}^{j} \cdot\left(\mathbf{E}_{0}+i \frac{k^{3}}{6 \pi \epsilon_{b}} \sum_{n} a_{m}^{n}(\omega) \mathbf{p}_{m}^{n}\right),
\end{aligned}
$$

which leads to

$$
a_{m}^{j}(\omega)=\left[\delta^{j n}-i \frac{f_{m}^{j}(\omega) k^{3}}{6 \pi \epsilon_{b}} \sum_{n} \mathbf{p}_{m}^{j} \cdot \mathbf{p}_{m}^{n}\right]^{-1} f_{m}^{j}(\omega) \mathbf{p}_{m}^{j} \cdot \mathbf{E}_{0} .
$$

As an example, Eq. (एव) for a particle with a single resonant mode can be written as

$$
a_{m}^{j} \approx \frac{-A_{m}^{j} \mathbf{p}_{m}^{j} \cdot \mathbf{E}_{0}}{\omega-\omega_{m}^{j}+i\left[\Gamma_{m}^{j} / 2+A_{m}^{j} k^{3} p_{m}^{j}{ }^{2} / 6 \pi \epsilon_{b}\right]}
$$

where Eq. (四) has been used for $f_{m}^{j}(\omega)$. This equation shows explicitly how the LSP resonance width increases with increasing radiation.

The excitation amplitude of the $j$-th resonance, as mentioned beforehand, is large for modes with a strong dipolar character. However, these modes also have associated strong radiation and therefore lead to large radiation damping according to the the denominator of the last equation which thus limit the near-field strength of the LSP (Sönnichsen et al., 20022; Wokaun et all, 1982).

\section{E. Properties of isolated metal nanoparticles}

The electrostatic approximation described above enables the prediction of useful properties of LSP resonances:

i) For very small nanoparticles where the incident electric field is constant over the nanoparticle surface, we observe that LSP modes with a zero dipole moment $\mathbf{p}_{m}^{k}=0$ are not excited (Eq. ([י)). Two examples are the second and fourth modes in Fig. 叉 with $\gamma_{1}^{2}=2.42$ and $\gamma_{1}^{4}=2.62$, which are predominantly quadrupolar in nature with very small dipole moments. In practise there is always a small variation of the electric field over the nanoparticle surface enabling these modes to be excited. However, the excitations are generally very weak and not usually observed unless the nanoparticles are large. Thus, we note that only LSP modes with non-zero dipole moments are usually excited (Yang et al., 2010).

ii) The LSP mode is not excited if the incident electric field is perpendicular to the LSP dipole moment whereby $\mathbf{p}_{m}^{j} \cdot \mathbf{E}_{0}=0$. This results in a dependence of the LSP mode on the polarization of the incident light. In particular, the electric field of the light radiated from the LSP has a polarization related to the direction of the dipole moment, as in the farfield term of Eq. (四). Using a vector identity it is possible to write $(\hat{r} \times \mathbf{p}) \times \hat{r}=\mathbf{p}-(\hat{r} \cdot \mathbf{p}) \hat{r}$. In a direction normal to the LSP dipole moment, the radiated field is $\mathbf{E}_{\text {rad }} \propto \mathbf{p}_{m}^{j}\left(\mathbf{p}_{m}^{j} \cdot \mathbf{E}_{0}\right)$ which can have a polarization different from the incident field $\mathbf{E}_{0}$. This is a useful property of metal nanoparticles with non-degenerate LSP modes that can be used to manipulate the polarization direction of a light wave (Kosako et all, 2010); Ming et al., 200.9).

It is worth noting that symmetric nanoparticles, such as cylinders and spheres, have multiple degenerate LSP modes with orthogonal dipole moments. A cylinder has two orthogonal modes across its diameter whereas a sphere has three (details on this last example are given in section (5]). For such structures it is necessary to include all the degenerate modes in the interaction, so that the radiated field is $\mathbf{E}_{r a d} \propto \sum_{j} \mathbf{p}_{m}^{j}\left(\mathbf{p}_{m}^{j} \cdot \mathbf{E}_{0}\right)$. For example, a sphere with all three LSP dipole moments $\mathbf{p}_{m}^{j}=\mathbf{p}_{m}^{1} \hat{x}^{j}$ having identical magnitudes but different orientations given by the orthogonal unit vectors $\hat{x}^{j}$, the field radiated by the LSPs $\mathbf{E}_{\text {rad }} \propto\left(p_{m}^{1}\right)^{2} \sum_{j=1}^{3} \hat{x}^{j}\left(\hat{x}^{j} \cdot \mathbf{E}_{0}\right)=\left(p_{m}^{1}\right)^{2} \mathbf{E}_{0}$ has a polarization parallel to the incident field, so that 
no polarization conversion arises.

iii) The phase $\phi$ of the LSP relative to the phase of the incident light depends on frequency according to $\tan \phi=-\Gamma_{m}^{k} / 2\left(\omega-\omega_{m}^{k}\right)$ which is obtained from the ratio of the imaginary to the real parts of Eq. (四). Well below resonance the phase is approximately zero $\phi \approx 0$; it passes through $90^{\circ}$ on resonance $\phi=\pi / 2$; and approaches $180^{\circ}$ above resonance $\phi \rightarrow \pi$. This is the characteristic behavior of a driven oscillator ubiquitous in physics. As we show below, the coupling between LSPs leads to coupled oscillators and frequency splitting, another property that is found in many different fields of physics.

\section{F. Coupled LSPs}

One useful property of LSPs is that they enable the manipulation of the phase and polarization of optical fields (Dregely et al., 2010; Ebbesen et al., 2008; Langguth et al., 2015; Liu et al., 2005). This can be achieved by placing two or more metal nanoparticles in close proximity such that the evanescent electric fields from the LSPs induce electric charges, and hence new LSPs, on neighboring particles (Gallinet et al., 2013; HaLas et al., 2010; Langguth et al., 2015). Traditionally, these coupling problems are difficult to solve and are usually analysed numerically (Brandl et al., 2006). However, we show below that the electrostatic eigenmode method leads to very simple expressions for many of the nanoparticle configurations of interest.

In the theory outlined above, the strength of a particular LSP resonance in an isolated nanoparticle is determined by the excitation amplitude $a_{m}^{k}$. If there are multiple nanoparticles, then the excitation amplitudes change. The electric field at the position of nanoparticle $m$ arises from two sources: the incident field $\mathbf{E}_{0}$ and the electric fields from the LSPs on all the other nanoparticles $\sum_{n} \mathbf{E}_{n}$. These extra fields must be included in the expression ([) for the excitation amplitude yielding a new amplitude $\tilde{a}_{m}^{k}(\omega)$ given by

$\tilde{a}_{m}^{k}(\omega)=f_{m}^{k}(\omega) \oint \tau_{m}^{k}\left(\mathbf{r}_{m}\right) \hat{n}_{m} \cdot\left(\mathbf{E}_{0}+\sum_{n} \mathbf{E}_{n}\left(\mathbf{r}_{m}\right)\right) d S_{m}$

where the integral is over nanoparticle $m$. The electric field $\mathbf{E}_{n}\left(\mathbf{r}_{m}\right)$ at particle $m$ arising from particle $n$ can be found from Coulomb's law. The total surface-charge associated with particle $n$ is $\sigma_{n}=\sum_{j} \tilde{a}_{n}^{j} \sigma_{n}^{j}$ so that

$$
\mathbf{E}_{n}\left(\mathbf{r}_{m}\right)=\frac{1}{4 \pi \epsilon_{b}} \sum_{j} \tilde{a}_{n}^{j}(\omega) \oint \sigma_{n}^{j}\left(\mathbf{r}_{n}\right) \frac{\hat{n}_{m} \cdot\left(\mathbf{r}_{m}-\mathbf{r}_{n}\right)}{\left|\mathbf{r}_{m}-\mathbf{r}_{n}\right|^{3}} d S_{n}
$$

Combining Eq. (ㅍ.5) with Eq. (피) and noting that the first term in Eq. (ㅍ) is just the excitation amplitude $a_{m}^{k}$ of nanoparticle $m$ when it is isolated, we have

$$
\tilde{a}_{m}^{k}(\omega)=a_{m}^{k}(\omega)+f_{m}^{k}(\omega) \sum_{n, j} \tilde{a}_{n}^{j} G_{m n}^{k j}
$$

where

$$
G_{m n}^{k j}=\frac{1}{4 \pi \epsilon_{b}} \oint \oint \tau_{m}^{k}\left(\mathbf{r}_{m}\right) \frac{\hat{n}_{m} \cdot\left(\vec{r}_{m}-\vec{r}_{n}\right)}{\left|\vec{r}_{m}-\vec{r}_{n}\right|^{3}} \sigma_{n}^{j}\left(\vec{r}_{n}\right) d S_{n} d S_{m},
$$

is the Coulomb coupling from nanoparticle $n$ to nanoparticle $m$. We define this coupling such that $G_{m m}^{k j}=0$. Equation (미) can be rearranged

$$
\sum_{n, j}\left(\delta_{m n} \delta^{k j}-f_{m}^{k}(\omega) G_{m n}^{k j}\right) \tilde{a}_{n}^{j}(\omega)=a_{m}^{k}(\omega),
$$

which has the form of a matrix equation that can be written in terms of an inverse

$$
\tilde{a}_{n}^{j}(\omega)=\sum_{m, k}\left(\delta_{m n} \delta^{k j}-f_{n}^{j}(\omega) G_{n m}^{j k}\right)^{-1} a_{m}^{k}(\omega) .
$$

This equation is extremely useful for describing the interaction between metal nanoparticles supporting LSP resonances. It relates the excitation $\tilde{a}_{n}^{j}(\omega)$ of the nanoparticle when it is a member of an ensemble to the excitations $a_{m}^{k}(\omega)$ of each of the isolated nanoparticles. We know the functional form of the single particle excitations - they are given by Eqs. (प) and (प). By inverting the matrix in Eq. (एप) we can determine what the excitations look like when the particles are coupled together.

The geometric coupling coefficient $G_{n m}^{j k}$ represents the interaction of the evanescent electric fields of the LSPs. Unless we need to calculate exact numerical values, it is not usually necessary to determine its magnitude in order to understand how metal nanoparticles behave when coupled together. However, it is useful to know whether or not this factor is positive or negative. A simplified expression is obtained by imagining two particles $n$ and $m$ separated by a distance $\mathbf{d}_{n m}$ sufficiently large that the integrals over the nanoparticle surfaces in Eq. (ए7) leave $\mathbf{r}_{n}-\mathbf{r}_{m}$ almost constant. We can write $\mathbf{r}_{n}-\mathbf{r}_{m}=\mathbf{s}_{n}-\mathbf{s}_{m}+\mathbf{d}_{n m}$ where $\mathbf{s}_{n}$ is a small vector pointing from the centre of nanoparticle $n$ to a point on its surface, and likewise for $\mathbf{s}_{m}$. Then the factor $\left|\mathbf{r}_{n}-\mathbf{r}_{m}\right|^{-3} \approx d_{n m}^{-3}\left(1-3\left(\mathbf{s}_{n}-\mathbf{s}_{m}\right) \cdot \hat{d}_{n m} / d_{n m}\right)$ can be expanded in a Taylor series and only the lowest order terms retained. When this expression is placed in Eq. (ㅍ) and all the multi-pole terms higher than dipole-dipole terms are ignored, we find that

$$
G_{m n}^{k j} \approx \frac{1}{4 \pi \epsilon_{b} d_{n m}^{3}}\left[3\left(\mathbf{p}_{m}^{k} \cdot \hat{d}_{n m}\right)\left(\mathbf{p}_{n}^{j} \cdot \hat{d}_{n m}\right)-\mathbf{p}_{m}^{k} \cdot \mathbf{p}_{n}^{j}\right]
$$

We recognise this expression as the interaction between two dipoles separated by a distance $d_{n m}$ where $\hat{d}_{n m}$ is the unit vector from particle $m$ to particle $n$. This shows that the coupling $G_{m n}^{k j}$ between two LSP modes is zero if the two modes have orthogonal dipole moments $\mathbf{p}_{m}^{k}$. $\mathbf{p}_{n}^{j}=0$ and if either of the modes is perpendicular to the direction of the line joining the two particles, $\mathbf{p}_{m}^{k} \cdot \hat{d}_{n m}=0$ or $\mathbf{p}_{n}^{j} \cdot \hat{d}_{n m}=0$. Equation (미) is useful in deciding whether or not two particles with LSPs will interact and 
whether or not that interaction is positive $G_{m n}^{k j}>0$ or negative $G_{m n}^{k j}<0$. Note that this coupling is symmetric under interchange of the nanoparticles: $G_{m n}^{k j}=G_{n m}^{j k}$. More generally we find that the coupling between particle pairs as described by Eq. (ए7) is symmetric unless the surface charge on one particle is concentrated at a point and the separation between particles is very small (Davis et al. $200.9 \mathrm{~b})$.

\section{ANALYSING INTERACTIONS BETWEEN COUPLED LSPS}

Now we apply the above theory to understand the interaction among ensembles of metal nanoparticles supporting localized surface plasmon resonances. There are four key equations that we use for this. They are:

i) the expression ([) for the LSP excitation of a single nanoparticle;

ii) the approximate expression (प) for the resonance factor;

iii) the matrix equation (एप) linking the coupled excitations $\tilde{a}_{n}^{j}$ to the uncoupled excitations $a_{m}^{k}$;

iv) the dipole approximation (201) for the coupling.

These equations reduce the coupling problem to relatively simple algebra. The resulting expressions can be analysed to understand the effects of coupling and the functional forms of the LSP excitations can be graphed to understand the frequency dependence.

In all cases shown below, we assume each isolated nanoparticle has a single LSP resonance that dominates at the frequency of the incident light so that we need only consider one LSP mode on each particle. In this case we can drop the mode index, so that terms like $G_{n m}^{11} \rightarrow G_{n m}$ and the equations are easier to follow. Also it is useful to write the denominator in Eq. (प) in short hand $\delta \tilde{\omega}_{n}=\omega-\omega_{n}+i \Gamma_{n} / 2$ which saves us from writing out resonances and the FWHM until the end. In this shorthand notation, resonances occur when the real part $\delta \tilde{\omega}_{n}=0$ is zero. In solving coupling problems it is useful to have algebra manipulation software that can invert the matrix (प्प) algebraically. In our case we use the software Derive which is freely available.

\section{A. Two particle coupling, dark modes and Fano resonances}

The simplest coupling problem is between two metal particles (Nordlander et al., 2004). To emphasise that the theory is not restricted to regular shapes, such as rods, disks or spheres, we show in Fig. B(a) the lowest order resonances of two random-shaped metal nanostructures. Although the LSP modes may show complicated multipole structures, the incident light couples predominantly to the dipole moments of the modes, as we discussed in the previous section.

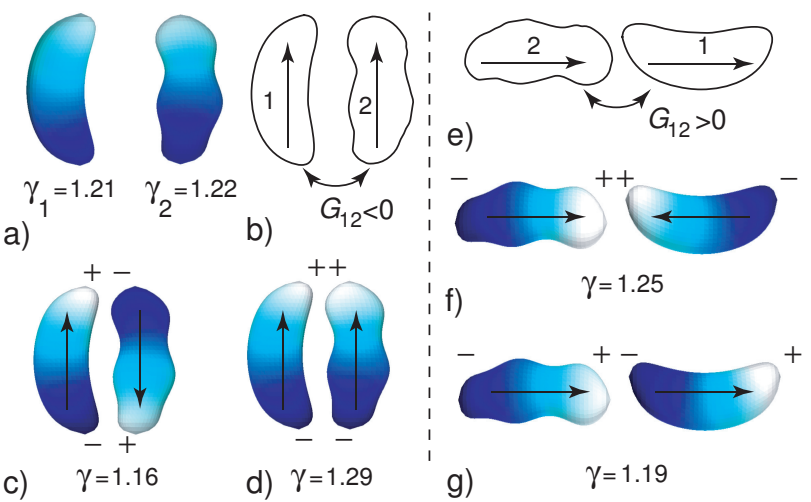

FIG. 3 An example of two coupled metal particles of arbitrary shape showing the formation of new resonant modes. a) the first LSP modes of the two shapes and their eigenvalues; b) a sketch used to calculate the side-by-side coupling; c) the 'dark' mode as determined numerically. The arrows show the dipole moments; d) the 'bright' mode as determined numerically; e) a sketch for end-to-end coupling; f) the 'dark' mode; h) the 'bright' mode. The signs show the relative surface charges on each of the modes.

To solve the coupling problem we number each nanostructure (in this case 1 and 2), guess at the directions of the dipole moments of the fundamental LSP modes (usually parallel to the major axis), draw the dipole moments as shown (Fig. B(b)), and write down the coupling between them. From Eq. (西) we note that the coupling $G_{12}=G_{21}<0$ is negative so we write $G_{12}=G_{21}=-G$, where $G$ is a positive quantity. Then, we apply Eq. ([प्Q) to form the interaction matrix

$$
\begin{aligned}
\left(\begin{array}{l}
\tilde{a}_{1} \\
\tilde{a}_{2}
\end{array}\right) & =\left(\begin{array}{cc}
1 & -f_{1} G_{12} \\
-f_{2} G_{21} & 1
\end{array}\right)^{-1}\left(\begin{array}{l}
a_{1} \\
a_{2}
\end{array}\right) \\
& =\frac{1}{\Delta}\left(\begin{array}{cc}
1 & f_{1} G_{12} \\
f_{2} G_{21} & 1
\end{array}\right)\left(\begin{array}{l}
a_{1} \\
a_{2}
\end{array}\right) .
\end{aligned}
$$

The matrix inverse is easily found and has a determinant $\Delta=1-f_{1} f_{2} G^{2}$ where we make the substitution $G_{12}=$ $G_{21}=-G$. The total dipole moment is given by $\mathbf{p}=$ $\tilde{a}_{1} \mathbf{p}_{1}+\tilde{a}_{2} \mathbf{p}_{2}$ where $\mathbf{p}_{1}$ and $\mathbf{p}_{2}$ are the eigenfunction dipole moments of the LSP modes.

For illustration purposes we will assume the LSP dipole moments are the same $\mathbf{p}_{1}=\mathbf{p}_{2}=\mathbf{p}_{\mathrm{LSP}}$, that the loss terms are the same $\Gamma_{1}=\Gamma_{2}=\Gamma$ but the resonance frequencies differ by an amount $2 \delta$ so that $\omega_{1}=\omega_{\mathrm{av}}-\delta$ and $\omega_{2}=\omega_{\mathrm{av}}+\delta$ where $\omega_{\mathrm{av}}$ is the average of the two resonance frequencies. Using the shorthand notation $\delta \tilde{\omega}=\omega-\omega_{\mathrm{av}}+i \Gamma / 2$ then from Eq. (可) we can write $f_{1}=$ $-A /(\delta \tilde{\omega}+\delta), f_{2}=-A /(\delta \tilde{\omega}-\delta)$. With Eq. (U) we have the uncoupled amplitudes $a_{1}=-A \mathbf{p}_{\mathrm{LSP}} \cdot \mathbf{E}_{0} /(\delta \tilde{\omega}+\delta)$ and $a_{2}=-A \mathbf{p}_{\mathrm{LSP}} \cdot \mathbf{E}_{0} /(\delta \tilde{\omega}-\delta)$ and from the matrix (खU) we can show that the amplitudes of the LSPs on 
each nanostructure are given by

$$
\begin{aligned}
& \tilde{a}_{1}=\frac{-A \mathbf{p}_{\mathrm{LSP}}\left(\mathbf{p}_{\mathrm{LSP}} \cdot \mathbf{E}_{0}\right)(\delta \tilde{\omega}-\delta+A G)}{(\delta \tilde{\omega}-g)(\delta \tilde{\omega}+g)} \\
& \tilde{a}_{2}=\frac{-A \mathbf{p}_{\mathrm{LSP}}\left(\mathbf{p}_{\mathrm{LSP}} \cdot \mathbf{E}_{0}\right)(\delta \tilde{\omega}+\delta+A G)}{(\delta \tilde{\omega}-g)(\delta \tilde{\omega}+g)}
\end{aligned}
$$

where $g=\left(\delta^{2}+A^{2} G^{2}\right)^{1 / 2}$. The total dipole moment of the coupled pair is then

$$
\mathbf{p}=\frac{-2 A \mathbf{p}_{\mathrm{LSP}}\left(\mathbf{p}_{\mathrm{LSP}} \cdot \mathbf{E}_{0}\right)(\delta \tilde{\omega}+A G)}{(\delta \tilde{\omega}-g)(\delta \tilde{\omega}+g)} .
$$

These equations show a number of important features found in coupled plasmonic systems. The easiest way to demonstrate these is to ignore the loss terms and set $\Gamma=$ 0 . Later we will include this again to plot the resonance spectra.

As an observation, note that the coupled amplitudes $\tilde{a}$ become large when the determinant $\Delta$ of the matrix in Eq. (एT) becomes small. The condition that the real part of $\Delta=0$ is zero generally determines the resonances of the coupled system. Moreover, the condition $\Re \Delta=0$ can be expressed as a polynomial in frequency. The order of the polynomial determines the number of solutions, which then determines the number of resonances. For example the determinant $\Delta=1-f_{1} f_{2} G^{2}$ from Eq. (四) has at most two solutions, so there are at most two resonances. In practise, as we show below, it is possible for one or more of the zeros to cancel corresponding terms in the numerator. Such resonances are then virtual in that they cannot be excited and can be associated with dark modes.

\section{Plasmon hybridization}

We take the simple situation of two nanostructures with the same resonance frequencies whereby $\delta=0$. If we look at the denominator in Eqs. ([2]) or ([2]) we see that it becomes zero when $\Re \delta \tilde{\omega}= \pm|A G|$ or $\omega=\omega_{R} \pm|A G|$. In this case the single resonance $\omega_{R}$ splits into two resonances, with a splitting that depends on the strength of the coupling $G$. Such frequency splitting is a common feature of coupled oscillators. In plasmonics literature, the formation of the new resonant modes, as in Fig. 3(c) \& (d), and the associated frequency splitting is often referred to as hybridization (Nordlander et al., [2004); Prodan and Nordlander, 2004; Prodan et al., 2003), in analogy with the description of the nature of chemical bonds in terms of the superpositions of atomic orbitals. In both cases, the hybridization leads to an antisymmetric mode (Fig. B(c)) and a symmetric mode (Fig. B(d)). The antisymmetric mode has opposite charges on the two metal structures facing each other, which is a lower energy configuration. The symmetric mode has the same charges facing one another creating a higher energy configuration. However, we note when $\delta=0$, one of the resonance terms in the denominator of Eq. (ㅍ.3) cancels with a similar term in the numerator. When $G>0$ the term responsible for the low frequency resonance cancels, $(\delta \tilde{\omega}+A G) /(\delta \tilde{\omega}+|A G|)=1$. In this case the low frequency resonance, or antisymmetric mode, has a zero net dipole moment and will not radiate light. This is a dark mode that we examine in more detail below. The remaining bright mode has an energy that varies depending on the coupling. When $G<0$, the high frequency resonance term cancels $(\delta \tilde{\omega}+A G) /(\delta \tilde{\omega}-|A G|)=1$ leaving only the low frequency resonance, which is still a bright mode.

The sign of the coupling can change based on the relative orientations of the nanostructures. If we had allowed the orientations of the nanostructures in Fig. 3 to vary, so that $\mathbf{p}_{n}=p_{\mathrm{LSP}}\left(\cos \theta_{n} \hat{x}+\sin \theta_{n} \hat{y}\right)$, then the coupling determined from Eq. (ख्य) takes the form $G=-G_{12} \propto-3 \cos \theta_{1} \cos \theta_{2}+\cos \left(\theta_{2}-\theta_{1}\right)$ where $\hat{d}_{12}=\hat{x}$ and $\theta_{1}$ is the angle nanostructure 1 takes with the $x$ axis and likewise for $\theta_{2}$ (Davis et al. 20100). In this situation the strength and sign of the coupling changes with the orientation of the nanostructures so that the energies, or resonance frequencies, of the hybrid modes change with orientation. For example, with $\theta_{1}=\theta_{2}=\pi / 2$ as shown in Fig. 3(b), it is the high frequency resonance that is the bright mode (Fig. B(d)). In this case the LSP resonance has a higher energy because the resonant mode has charges of the same sign opposite one another. With $\theta_{1}=\theta_{2}=0$ so that the nanostructures are aligned end to end (Fig. B(e)), $G<0$ and it is the low frequency resonance that is the bright mode (Fig. 31(g)) and the nanostructures have opposite charges facing one another, thus reducing the overall energy of the mode. The shifts in the plasmon resonances with coupling have been observed experimentally, with red shifts observed for end-to-end coupling (Finston et al., [20019; Haynes and Van Duyne, 2001]; Jain et al, 20106; Rechberger et al., 20033; Su et al., 20033; Thaxton and Mirkin, 2005) and blue shifts for side-by-side coupling (Funston et al. [200.9; Jain et al., 2006; Rechberger et al., 20013) consistent with our analysis above.

\section{Bright and dark modes}

Suppose the coupling is strong so that $\delta \ll A G$ which implies that $g \approx A G$. At the low frequency resonance $\delta \tilde{\omega}=-g \approx-A G$ then the numerator of the LSP resonance $\tilde{a}_{1}$ in Eq. ([2Z) is $\delta \tilde{\omega}-\delta+A G \approx-\delta$ whereas the numerator for particle 2 is $\delta \tilde{\omega}+\delta+A G \approx \delta$ which has the opposite sign to $\tilde{a}_{1}$. In effect, the direction of the dipole moment on one of the particles is opposite to the way we have drawn it for this LSP mode. This describes a resonance mode in which the LSPs on the two nanostructures have dipole moments pointing in opposite directions and therefore are oscillating $180^{\circ}$ out of phase (Fig. B(c)). On the other hand, at the higher frequency resonance $\delta \tilde{\omega}=g \approx A G$ then both amplitudes have the same sign. For this resonance the LSPs oscillate in phase. This be- 
haviour agrees with the numerical simulation shown in Fig.3(c), (d). When we look at the total dipole moment we see that when $\delta \tilde{\omega}=-g \approx-A G$ the dipole moment Eq. (ㄹ.3) is zero so there is no radiation from the oscillating LSPs (when we include losses such that $\Gamma \neq 0$ there is a small residual dipole moment). This is a "dark mode" and is a consequence of the two LSPs oscillating out of phase. Provided $\delta \neq 0$ the incident light can excite this mode but there is little radiation from it and therefore little scattering and radiative damping.

\section{Fano resonances}

The total dipole moment of the coupled LSP system has a term in the numerator that is linearly dependent on the frequency, and its real part goes to zero at some frequency. This particular mathematical form is characteristic of a Fano resonance (Fano, 196]; Luk'yanchuk et al., 2010; Mirin et al. 2, 20019; Miroshnichenko et al.., 20110). The Fano resonance arises from the interference between an incident wave and a wave scattered from a resonant object (Lovera et al., [2013). There are three conditions for the phase at which a resonant object oscillates, depending on the frequency of the applied driving force: (1) It oscillates in phase with the driving force when driven well below its resonance frequency, (2) it oscillates 90 degrees out of phase when resonantly driven, and (3) it oscillates 180 degrees out of phase when driven well above resonance. If the resonance oscillation of the object is responsible for re-emitting (or scattering) a wave, then the interference of the incident wave with the scattered one can alternate between destructive and constructive, resulting in a characteristic asymmetric resonance spectrum. This asymmetric Fano profile has the form $I=A_{F}\left(E_{\omega}+q\right)^{2} /\left(E_{\omega}^{2}+1\right)$ where $q$ is a "shape factor" and $E_{\omega}$ represents a frequency-dependent normalised energy term. The lineshape is asymmetric around a minimum, which occurs at a frequency $E_{\omega}=-q$ arising from the interference between two components of the system.

The intensity of the scattered radiation from the coupled nanorods is proportional to $|\mathbf{p}|^{2}$, which according to Eq. ([2:3) has the approximate form of a Fano resonance (around the lower frequency resonance). Fig. 田 shows a plot of the intensity, where the almost-dark mode demonstrates an asymmetric profile that can be fitted by the Fano equation. The Fano profile in this case arises from the interference of the radiation from two nanorods with different resonance frequencies, leading to constructive or destructive interference depending on the applied frequency.

\section{B. Coupling in three nanorod structures}

In this section we analyse structures consisting of three nanorods coupled together. We begin with a simple

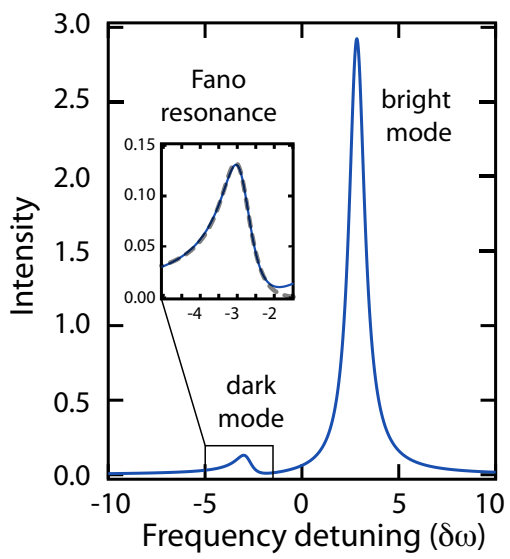

FIG. 4 A Fano resonance associated with two coupled metal particles. The intensity is calculated as a function of detuning $\delta \omega=\omega-\omega_{R}$ from the centre resonance based on $|\mathbf{p}|^{2}$ from Eq. ([2.3) with $\left|2 A \mathbf{p}_{1}\left(\mathbf{p}_{1} \cdot \mathbf{E}_{0}\right)\right|=1, A G=2, \Gamma=1$ and $\delta=2$. The inset shows the dark mode and the dashed curve is the Fano expression with the scaled energy term $E_{\omega}=2(\delta \omega / \Gamma+$ 2.87), $q=-3.5$, and an amplitude scaling factor $A_{F}=0.01$ (see Miroshnichenko et al. (2010) discussion around their Eqn. (1))

structure that demonstrates a variety of different properties, such as "induced" transparency, phase sensitivity, all-optical modulation and switching, and then discuss a radially symmetric structure that demonstrates radial dark modes.

As shown in Fig. 圆, we consider a set of three nanorods forming a "dolmen"-like structure. We assume the two parallel nanorods are sufficiently far apart that the interaction between them is dominated by their coupling to nanorod 3. We also assume that all three nanorods are identical and therefore have the same LSP resonance frequency $\omega_{R}$, loss terms $\Gamma$ and eigenfunction dipole moments $\mathbf{p}_{1}=\mathbf{p}_{2}=\mathbf{p}_{3}$. Under these conditions, Eq. (미) describing the excitation amplitudes for these coupled nanorods is given by

$$
\begin{aligned}
\left(\begin{array}{c}
\tilde{a}_{1} \\
\tilde{a}_{2} \\
\tilde{a}_{3}
\end{array}\right) & =\left(\begin{array}{ccc}
1 & 0 & -f G \\
0 & 1 & f G \\
-f G & f G & 1
\end{array}\right)^{-1}\left(\begin{array}{c}
a_{1} \\
a_{2} \\
a_{3}
\end{array}\right) \\
& =\frac{1}{\Delta}\left(\begin{array}{ccc}
1-f^{2} G^{2} & -f^{2} G^{2} & f G \\
-f^{2} G^{2} & 1-f^{2} G^{2} & -f G \\
f G & -f G & 1
\end{array}\right)\left(\begin{array}{l}
a_{1} \\
a_{2} \\
a_{3}
\end{array}\right)
\end{aligned}
$$

where $G>0$ represents the coupling between the particles and $\Delta=1-2 f^{2} G^{2}$ is the matrix determinant. Having inverted the matrix we obtain in just a few lines of algebra the fundamental equations that describe the interactions among these three nanorods and an incident field, as we now proceed to illustrate. 


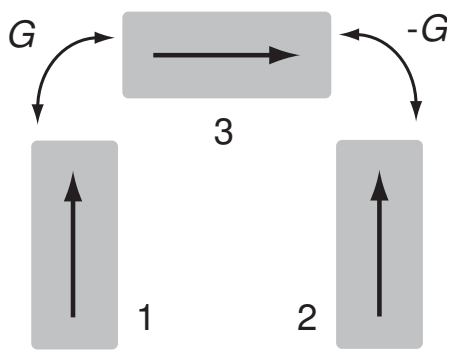

FIG. 5 A three-particle plasmonic structure that mimics the Wheatstone bridge in electronics (Davis et al., 2009b). The arrows represent the dipole moments of the fundamental LSP modes and $G$ is the coupling coefficient.

\section{Plasmon-induced transparency}

For this example the incident light is polarized parallel to nanorod 3 so that $a_{1}=a_{2}=0$ (this also mimics the experimental conditions of Liu et al. (2009)). With Eq. (ख4), it is straightforward to show that $\tilde{a}_{1}=f G \tilde{a}_{3}$ and $\tilde{a}_{2}=-f G \tilde{a}_{3}$, that is, these two rods oscillate out-ofphase with each other resembling a collective quadrupole (dark) mode (Liu et al., 2000. ). Since $\tilde{a}_{1}+\tilde{a}_{2}=0$, the total dipole moment in the polarisation along these two rods vanishes, and this reduces radiative damping, as in Eq. (피).

The light scattered from nanoparticle 3 is described by the excitation amplitude $\tilde{a}_{3}$, which according to Eq. (24) is given by $\tilde{a}_{3}=a_{3} /\left(1-2 f^{2} G^{2}\right)$. Using the shorthand notation $\delta \tilde{\omega}=\omega-\omega_{R}+i \Gamma / 2$, we write $a_{3}=-A \mathbf{p}_{3} \cdot \mathbf{E}_{0} / \delta \tilde{\omega}$ and $f=-A / \delta \tilde{\omega}$ which leads to

$$
\tilde{a}_{3}=\frac{-A\left(\mathbf{p}_{3} \cdot \mathbf{E}_{0}\right) \delta \tilde{\omega}}{(\delta \tilde{\omega}-\sqrt{2} A G)(\delta \tilde{\omega}+\sqrt{2} A G)} .
$$

We immediately see that the coupling of nanorod 3 to the two parallel nanorods leads to a resonance splitting that depends on the coupling strength $G$. The two new resonances occur at frequencies $\omega_{+}$for which the denominator of Eq. (2.5) is zero, that is when $(\delta \tilde{\omega})^{2}+2(A G)^{2}=0$. For weak coupling, $G \approx 0$ and there is only one resonance that occurs at $\omega_{R}$ (the resonance frequency of the individual particle). As the coupling increases, the resonances $\omega_{ \pm}$move apart (Fig. [6), and the magnitude of the excitation amplitude $\tilde{a}_{3}$ decreases significantly at zero detuning (i.e when the real part of $\delta \tilde{\omega}=0$ ). This strong drop in the excitation amplitude leads to a dramatically reduced scattering of the light at the frequency of the isolated nanoparticle $\left(\omega_{R}\right)$, which is experimentally observable as an increase in the transparency of an array of such structures (Hokari et al., 2014); Liu et al., 200.9). This effect has been termed plasmon-induced "transparency" (Zhang et al., 2008) and is related to the splitting of the resonances due to coupling. If we had included the radiation damping in Fig. [6, then the width of the transparency region would be narrower than shown, due to the reduced damping associated with the quadrupole.

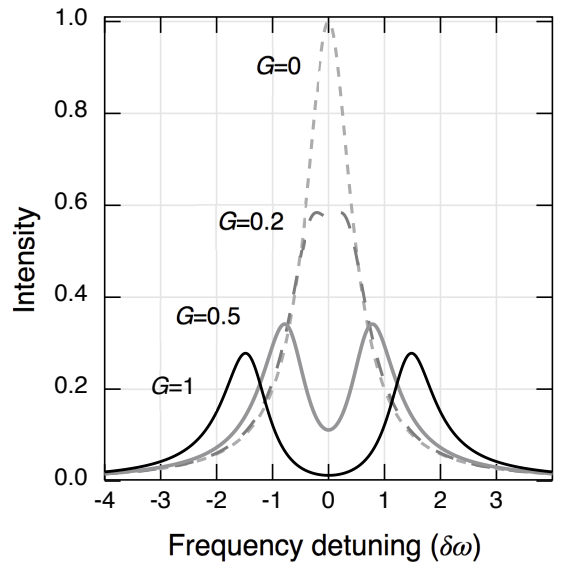

FIG. 6 The spectrum of a three-particle configuration that demonstrates the formation of induced transparency at zero detuning $\delta \omega=\omega-\omega_{R}$ as the coupling strength $G$ increases. The spectra are based on $\left|\tilde{a}_{3}\right|^{2}$ calculated from Eq. ([25) with $\Gamma=1, A=1, \mathbf{p}_{3} \cdot \mathbf{E}_{0}=0.5$

\section{Plasmonic Wheatstone bridge}

Suppose instead we excite the structure in Fig. with light polarized parallel to the two nanorods $(1 \&$ 2 ) and perpendicular to the third. However, we place a polariser at the output that only accepts light radiated from nanorod 3. In this case $a_{1} \neq 0$ and $a_{2} \neq 0$ but $a_{3}=0$ and according to Eq. (ख4), the output $\tilde{a}_{3}=f G\left(a_{1}-a_{2}\right) /\left(1-2 f^{2} G^{2}\right)$ now depends on the difference of the LSP excitations on the two parallel nanorods. Suppose we have light incident at some angle so there is a phase difference between the excitations on nanorods 1 and 2. That is, let $a_{1}=-A \mathbf{p}_{3} \cdot \mathbf{E}_{0} \exp (i \phi / 2) / \delta \tilde{\omega}$ and $a_{2}=-A \mathbf{p}_{3} \cdot \mathbf{E}_{0} \exp (-i \phi / 2) / \delta \tilde{\omega}$, then the LSP excited in nanorod 3 is given by

$$
\tilde{a}_{3}=\frac{2 i \sin (\phi / 2) A^{2} G\left(\mathbf{p}_{3} \cdot \mathbf{E}_{0}\right)}{(\delta \tilde{\omega}-\sqrt{2} A G)(\delta \tilde{\omega}+\sqrt{2} A G)} .
$$

The structure produces an LSP in the third rod with an amplitude that depends on the phase difference $\phi$ of the light incident on the two parallel rods. The configuration leads to the interference between the two surface plasmons on the parallel rods and, in effect, performs a mathematical difference operation on them. This configuration is analogous to the Wheatstone bridge circuit in electronics (Davis et al., 2009a) that generates a signal between its two arms when they are imbalanced. Nanorod 3 plays the role of the voltmeter that reads out the difference signal. Such structures can be extremely small and will probe optical phase within a fraction of a wavelength (Eftekhari et al., 2014), as shown in Fig. 7. The intensity of the scattered light is proportional to $\left|\tilde{a}_{3}\right|^{2}$, which varies with phase as $\sin ^{2}(\phi / 2) \approx \phi^{2} / 4$. This variation is quadratic in phase for small phase differences, in agreement with the experimental results. 

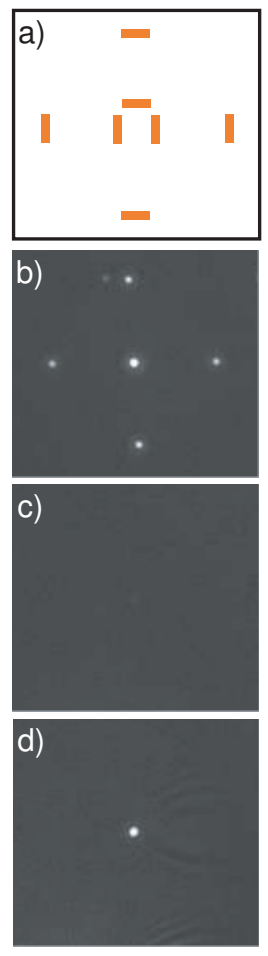

e)

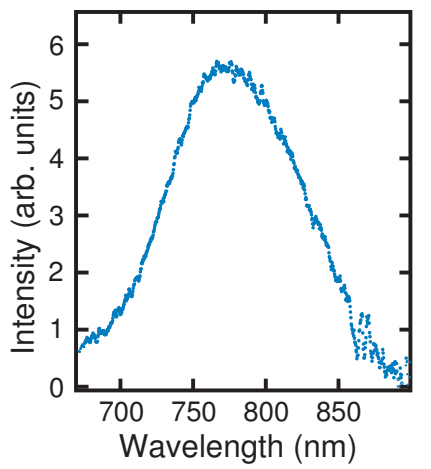

f)

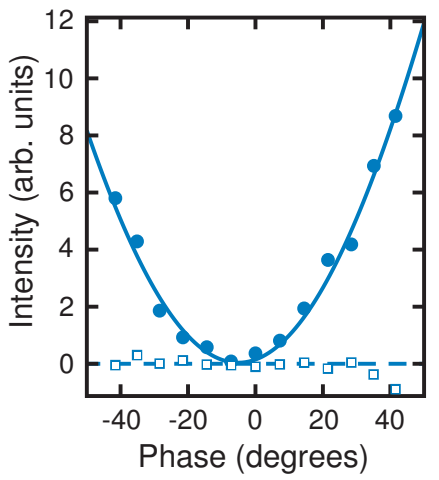

FIG. 7 Results of experiments on a single plasmonic Wheatstone bridge circuit. a) A schematic diagram of the configuration of nanorods, including four alignment rods used to ensure correct alignment of polarizers; b) a microscope image showing light scattered from the circuit and the four alignment nanorods; c) the scattering intensity obtained with the substrate correctly aligned and with normal incident light (zero phase difference); d) with light incident at $\theta=30^{\circ}$ the circuit appears as a bright spot at the centre of the image; e) scattering spectrum from the plasmonic circuit; f) the intensity as a function of phase difference between the two inputs (dots are experimental data, the solid curve is theory). Also shown is the null-response of the circuit (square points) where the phase change is between the parallel rods and rod 3. The dashed line is the expected response in the null orientation. Adapted from (Ettekhari et al., [2014)

\section{All-optical modulation}

In the last section we used an analogy between metal particles coupled by evanescent fields and an electric circuit operating at optical frequencies. This concept has been applied for some time to plasmonic structures, since the LSP resonance can be modelled by a combination of an inductor, capacitor and a resistor (Abasahl et al., 2014; Davis et al., 20119a; Engheta, 20107; Engheta et al., 20(15); Liu et al., 2013); Sun et al., 2012). Electrical circuits made from these components are linear, which means the circuit can be driven in reverse: that is, the outputs can be driven by a signal and an inverse response observed at the inputs. Since the plasmonic circuit in the previous example produces an output depending on the phase at the inputs, it should be possible to drive the output with an intensity that modulates the phase at the inputs.
In this final example using the same three-nanostructure configuration, we show that indeed this is possible by analysing what happens when light is incident on all three nanorods.

It is convenient to show the phases of all quantities explicitly. We express the resonance factor as a magnitude $f_{\omega}$ and a phase $\phi_{w}$ such that $f(\omega)=f_{\omega} e^{i \phi_{\omega}}$. Light is incident at some angle on two identical rods $1 \& 2$ lying in the $x-y$ plane at $z=0$ (Fig. $\mathbf{\nabla}(\mathrm{a})$ ). The light is polarised parallel to their long axes so that it does not

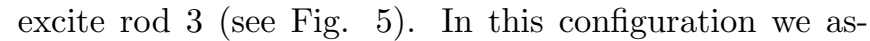
sume there is a polariser on the output that only passes light scattered from rods $1 \& 2$. With $\mathbf{k}_{I}$ the incident wavevector, there is a phase difference between the LSP excitations related to the vector separation $\mathbf{d}$. The phase difference is $e^{\mp i \mathbf{k}_{I} \cdot \mathbf{d} / 2}$. Likewise, light radiated from these rods in the same direction $\mathbf{k}_{I}$ has the opposite phase difference $e^{ \pm i \mathbf{k}_{I} \cdot \mathbf{d} / 2}$. Since the rods are identical, we can write $a_{1}=f_{\omega} S e^{-i \mathbf{k}_{I} \cdot \mathbf{d} / 2+i \phi_{\omega}}$ and $a_{2}=f_{\omega} S e^{i \mathbf{k}_{I} \cdot \mathbf{d} / 2+i \phi_{\omega}}$ where $S$ is proportional to the amplitude of the incident light.

There is also a second light beam incident that is polarised so that it only induces LSPs in rod 3. We impose a phase $\phi_{c}$ on this beam relative to the light incident on rods $1 \& 2$. Then $a_{3}=f_{\omega} C e^{i \phi_{c}+i \phi_{\omega}}$ where $C$ is proportional to the amplitude of the light. As a further approximation, we assume the coupling $G$ is small enough that we can neglect terms $G^{2}$ and higher. Then the amplitudes of the LSPs in rods $1 \& 2$ are obtained from the coupling matrix ([4])

$$
\begin{aligned}
& \tilde{a}_{1} \approx f_{\omega} e^{i \phi_{\omega}}\left(S e^{-i \mathbf{k}_{I} \cdot \mathbf{d} / 2}+G C f_{\omega} e^{i \phi_{c}+i \phi_{\omega}}\right) \\
& \tilde{a}_{2} \approx f_{\omega} e^{i \phi_{\omega}}\left(S e^{i \mathbf{k}_{I} \cdot \mathbf{d} / 2}-G C f_{\omega} e^{i \phi_{c}+i \phi_{\omega}}\right)
\end{aligned}
$$

Taking account of the fact that the two rods are separated by a small distance, then the scattered wave amplitude in direction $\mathbf{k}_{I}$ is

$$
\begin{aligned}
\tilde{a}_{\mathrm{T}} & =\tilde{a}_{1} e^{i \mathbf{k}_{I} \cdot \mathbf{d} / 2}+\tilde{a}_{2} e^{-i \mathbf{k}_{I} \cdot \mathbf{d} / 2} \\
& \approx 2 f_{\omega} e^{i \phi_{\omega}}\left[S+i G C f_{\omega} e^{i \phi_{c}+i \phi_{\omega}} \sin \left(\mathbf{k}_{I} \cdot \mathbf{d} / 2\right)\right],
\end{aligned}
$$

where any scattering from rod 3 is filtered out by a polariser. The intensity of the scattered wave is related to $I=\left|\tilde{a}_{\mathrm{T}}\right|^{2}$ which is

$$
\begin{gathered}
I=4 f_{\omega}^{2}\left[S^{2}-2 f_{\omega} G C S \sin \left(\mathbf{k}_{I} \cdot \mathbf{d} / 2\right) \sin \left(\phi_{c}+\phi_{\omega}\right)\right. \\
\left.+f_{\omega}^{2} G^{2} C^{2} \sin ^{2}\left(\mathbf{k}_{I} \cdot \mathbf{d} / 2\right)\right] .
\end{gathered}
$$

This equation shows the intensity $I$ of the beam scattered by an array of such structures modulated by the amplitude $C$ and phase $\phi_{c}$ of a beam of light incident on rod 3, even though this beam is filtered out by the polariser and does not reach the detector. The modulation arises due to interference between the LSPs created in the coupled structure by the two light beams.

The optical modulation strength $M$ can be defined as the difference of the intensity of the transmitted light 
when the phase is shifted from $\phi_{c}$ to $\phi_{c}+180^{\circ}$, divided by the intensity $4 f_{\omega}^{2} S^{2}$ of the beam scattered directly by rods $1 \& 2$. This gives

$$
M=4 f_{\omega} G(C / S) \sin \left(\mathbf{k}_{I} \cdot \mathbf{d} / 2\right) \sin \left(\phi_{c}+\phi_{\omega}\right) .
$$

a)
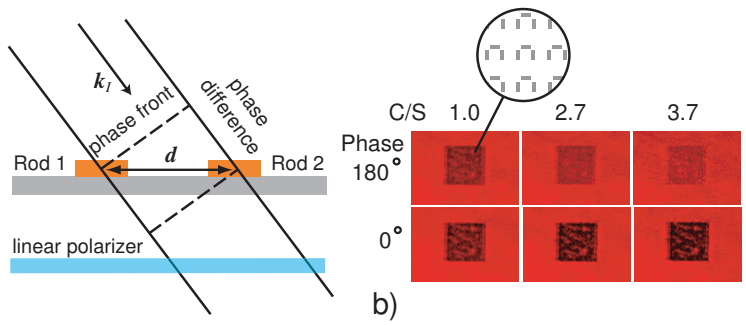

b)
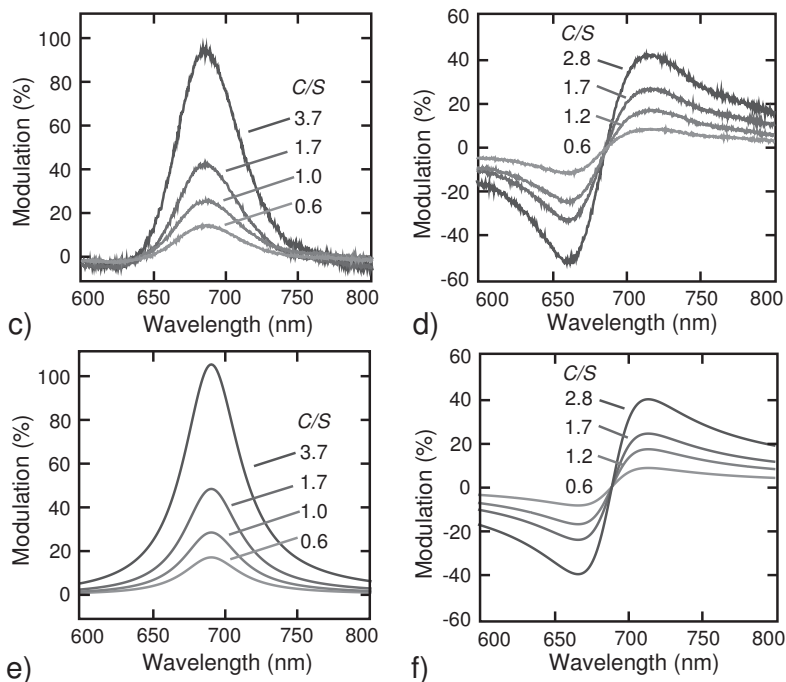

d)

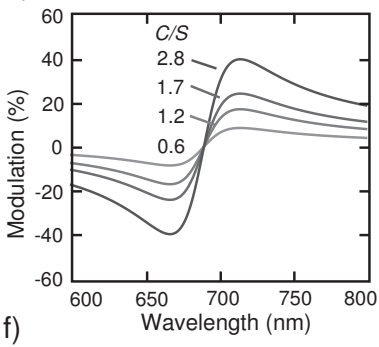

FIG. 8 A plasmonic all-optical modulator. A metamaterial can be created with arrayed plasmonic Wheatstone-bridge circuits (based on the diagram of Fig. 5) where a signal $S$ and a control $C$ beam are orthogonally polarized. a) Diagram showing the phase difference of LSP excitation on rods $1 \& 2$ on a substrate arising from a light beam incident at an angle; b) Images of the transmission through arrays of plasmonic circuits for different ratios $C / S$ and phases $\phi_{c}$, showing the modulation effect; c-d) Experimental measurements of the modulation determined for changes in phase c) $\phi_{c}=0^{\circ} \rightarrow 180^{\circ}$ d) $\phi_{c}=-90^{\circ} \rightarrow 90^{\circ}$; e-f) the corresponding theoretical modulation based on Eq. (30). Adapted from (1)avis et al., 20144)

In Fig. $8(\mathrm{~b})$ we show an experimental demonstration (Davis et al., 2014) of this all-optical modulation with a metamaterial created from an array of plasmonic Wheatstone-bridge circuits of Fig. 回. The transmission through the metamaterial changes when either the amplitude or phase of the "control" beam exciting nanorod 3 is varied. The experimentally measured modulations as functions of wavelength and control beam parameters are compared in Fig. 8(c)-(f) with calculations based on Eq. (\$तI). The resonance factor is given by $f_{\omega}=A /\left(\delta \omega^{2}+\Gamma^{2} / 4\right)$ and $\tan \phi_{\omega}=-\Gamma / 2 \delta \omega$. The resonance frequency and width used in the calculation was obtained from the experimental data and the unknown scale factors $4 A G$ were found by matching the modulation in Fig. $8(\mathrm{f})$ to the experimental value at $C / S=1.2$ and $\lambda=715 \mathrm{~nm}$. No other scaling was applied. Despite the complexity of this configuration and experiment, the formula (301) shows remarkable agreement with experiment, which highlights the predictive power of this simple coupling theory, despite the approximations that have been used in deriving it.

All-optical modulation in this structure originates by interfering the incident beam with a given polarisation by another beam with orthogonal polarisation in such a way that the second beam is unable to propagate through the metamaterial effectively. In one sense, the beam incident on nanorod 3 excites LSPs on nanorods $1 \& 2$ that oscillate $180^{\circ}$ out of phase with each other and therefore the beams scattered from these rods cancel out. However, the beam coupling from rod 3 interferes with the incident light scattered from rods $1 \& 2$ leading to a decrease in intensity. This can be thought of as a subwavelength interferometer. By making a diffraction grating based on these structures it is possible to create an alloptical switch, or a diffraction grating with an opticallycontrolled blaze (Davis et al., 2014).

\section{Radially-symmetric structures and dark modes}

Here we consider another three nanorod structure, one that exhibits radially-symmetric dark modes (Gómez et al. 2013), that we analyse using the theory developed in section 미.
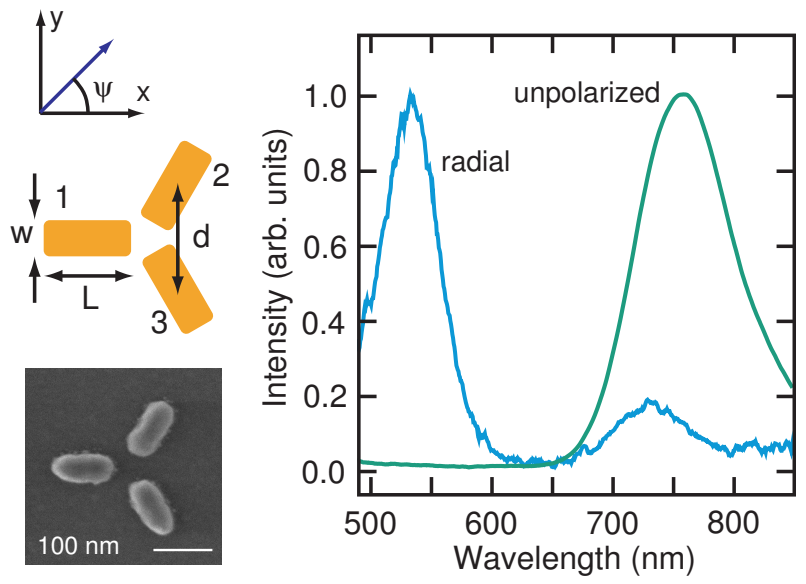

FIG. 9 Radially-symmetric trimer exhibiting a radial dark mode. Experimentally measured spectra for unpolarized and radially polarised light incident on a gold single trimer, shown in the SEM on the left. Adapted from Gómez et al. (2013)

To describe the interaction of light with the structure

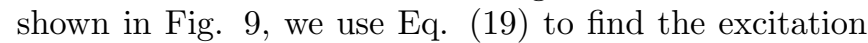
amplitudes under the assumption that all the particles are identical and equidistant. The matrix equation is 
(Gómez et al., 2013):

$$
\begin{aligned}
& \left(\begin{array}{l}
\tilde{a}_{1} \\
\tilde{a}_{2} \\
\tilde{a}_{3}
\end{array}\right)=\left(\begin{array}{ccc}
1 & -f G & -f G \\
-f G & 1 & -f G \\
-f G & -f G & 1
\end{array}\right)^{-1}\left(\begin{array}{l}
a_{1} \\
a_{2} \\
a_{3}
\end{array}\right) \\
& =\frac{1}{\Delta}\left(\begin{array}{ccc}
1-f^{2} G^{2} & f^{2} G^{2}+f G & f^{2} G^{2}+f G \\
f^{2} G^{2}+f G & 1-f^{2} G^{2} & f^{2} G^{2}+f G \\
f^{2} G^{2}+f G & f^{2} G^{2}+f G & 1-f^{2} G^{2}
\end{array}\right)\left(\begin{array}{l}
a_{1} \\
a_{2} \\
a_{3}
\end{array}\right)
\end{aligned}
$$

where the determinant $\Delta=1-2(f G)^{3}-3(f G)^{2}$ is of order 3 in $f$, which implies that the structure has three collective resonances.

To proceed further, we write the incident electric field $\mathbf{E}_{0}$ as a superposition of a linearly polarized component with amplitude $E_{l}$ and a radially polarized one with amplitude $E_{r}: \mathbf{E}_{0}=E_{l}(\cos \psi \hat{x}+\sin \psi \hat{y})+E_{r} \hat{r}$, where $\hat{r}$ is a unit vector pointing radially outwards from the origin of coordinates and $\psi$ is the angle that the linearly-polarised component subtends with respect to the horizontal. This is an approximate way to describe a field with radial symmetry. Accurate descriptions are more complicated as shown in the literature (Mojarad and Agio, 20019; SanchoParramon and Bosch, (2012). However, as we will show below, this simplistic approach allow us to extract physically relevant information that closely reproduces the experimental observations.

For the case of incident radial polarization $\left(E_{l}=0\right)$, straightforward (but lengthy) algebraic manipulation of Eq. (ㅍ) yields equal excitation amplitudes for all three nanorods, given by

$$
\tilde{a}=\frac{A E_{r} p}{\delta \tilde{\omega}-2 A G},
$$

which predicts the excitation of a radially-symmetric mode with net zero dipole moment and a resonance frequency given by the real part of $\omega_{d}=\omega_{R}+2 A G$.

For the case of linearly polarized light $\left(E_{r}=0\right)$ incident with a polarization angle $\psi$ as shown in Fig. Q, the excitation amplitudes are given by:

$$
\begin{aligned}
& \tilde{a}_{1}=-\frac{A E_{l} p}{A G+\delta \tilde{\omega}} \cos \psi \\
& \tilde{a}_{2}=\frac{A E_{l} p}{A G+\delta \tilde{\omega}} \frac{1}{2}(\sqrt{3} \sin \psi+\cos \psi) \\
& \tilde{a}_{2}=\frac{A E_{l} p}{A G+\delta \tilde{\omega}} \frac{1}{2}(\cos \psi-\sqrt{3} \sin \psi),
\end{aligned}
$$

and therefore the total dipole moment $\mathbf{p}=\sum_{n} \tilde{a}_{n} \vec{p}_{n}$ is

$$
\mathbf{p}_{l}=-\frac{3 A E_{l} p^{2}}{2(A G+\delta \tilde{\omega})}(\cos \psi \hat{x}+\sin \psi \hat{y})
$$

This equation represents a bright plasmon mode, with a doubly degenerate resonance frequency $\omega_{b}=\omega_{R}+A G$ red-shifted from the plasmon resonance of the individual nanorods.
The lineshape of the scattering spectrum of this $D_{3 h^{-}}$ symmetric trimer is proportional to $|\mathbf{p}|^{2}$, which according to Eq. (34) results in a single scattering band centred at the resonance frequency of the doubly-degenerate bright modes. According to Eq. (B2), the dark mode can be excited by a radially polarised beam of light (i.e. it can absorb), but by virtue of not having a net dipole moment, the absence of radiative damping leads to a narrower lineshape when compared to the bright mode (compare with Eq. (피)). These predictions were experimentally confirmed by Gómez et al. (2013) and summarised in figure $\mathbf{q}$ demonstrating here the predicting power of the analytic theory of section $\mathbb{W}$.

\section{Coupling in 3D structures}

We have shown the applicability of the theory developed in section $\mathbb{\square}$ to LSP coupling between two particles and among three particles. We now turn our attention to a special case consisting of the three-dimensional arrangement of Fig. سu, dubbed the three-dimensional plasmonic ruler a concept first introduced by Lin et al. (201I). This structure contains two vertically displaced metal nanorods dimers (aligned side to side) and a fifth nanorod, placed centrally at a right angle with respect to the dimers.

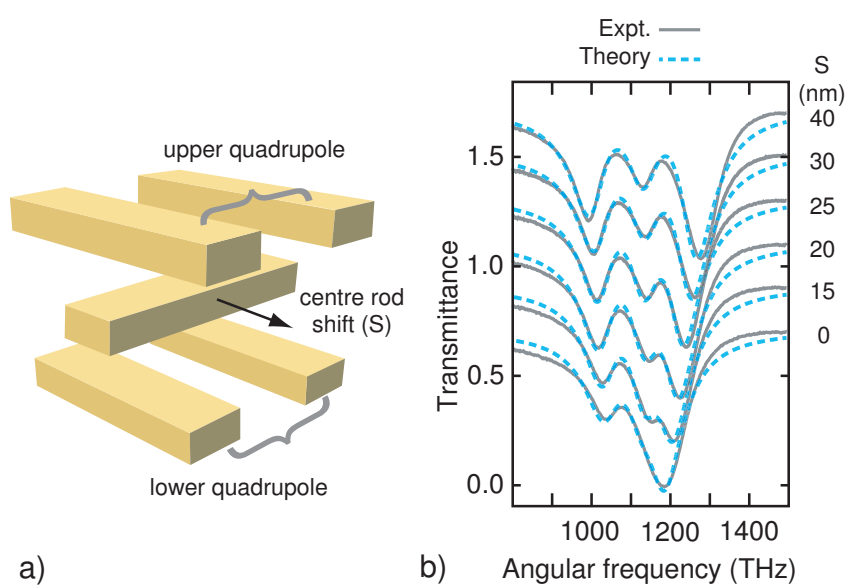

FIG. 10 Three-dimensional plasmonic ruler. a) A sketch of the ruler: the upper and lower quadrupoles are created by LSP dipole modes excited in opposite directions in each pair of rods due to coupling from the central rod; b) experimental data from Liu et al. (Liu et al. , 201U) and the analytical model fitted to the data for different lateral translations $\mathrm{S}$ of the central rod. Adapted from (ए)avis et al., [20ा2)

The symmetry of the three-dimensional plasmon ruler allows for significant simplifications of equation (피). For instance, the LSP coupling in each nanorod pair can be described with a single coupling constant, which for the sake of brevity we denote as $C_{n m} \equiv f_{n} G_{n m}$ (where $n$ and $m$ are particle indices). These pairs are not mutually coupled (by virtue of their long vertical separation distance), implying there are two $2 \times 2$ blocks describing 
LSP coupling for each pair and two $2 \times 2$ off-diagonal blocks containing only zeroes. Coupling between the central rod and the lower pair of nanorods are approximately equal but opposite, $C_{a 1}=-C_{a 2}$. Similar considerations for the remaining interactions involving the central rod lead to the following form for Eq. ([प])

$$
\left(\begin{array}{l}
\tilde{a}_{a} \\
\tilde{a}_{1} \\
\tilde{a}_{2} \\
\tilde{a}_{3} \\
\tilde{a}_{4}
\end{array}\right)=\left(\begin{array}{ccccc}
1 & -C_{a 1} & C_{a 1} & -C_{a 3} & C_{a 3} \\
-C_{a 1} & 1 & -C_{12} & 0 & 0 \\
C_{1 a} & -C_{12} & 1 & 0 & 0 \\
-C_{3 a} & 0 & 0 & 1 & -C_{34} \\
C_{3 a} & 0 & 0 & -C_{34} & 1
\end{array}\right)^{-1} \cdot\left(\begin{array}{l}
a_{a} \\
a_{1} \\
a_{2} \\
a_{3} \\
a_{4}
\end{array}\right) .
$$

The determinant of the matrix, required for the matrix inverse, is given by

$$
\begin{aligned}
\Delta & =\frac{2 G_{a l}^{2}}{\omega-\omega_{q l}+i \Gamma / 2}+\frac{2 G_{a u}^{2}}{\omega-\omega_{q u}+i \Gamma / 2} \\
& -\left(\omega-\omega_{a}+i \Gamma / 2\right),
\end{aligned}
$$

where $\omega_{q l}=\omega_{l}+G_{l}$ is the quadrupole resonance associated with the coupling $G_{l}$ between the lower pair of nanorods, and likewise $\omega_{q u}=\omega_{u}+G_{u}$ is the quadrupole resonance for the upper pair. The transmission spectrum is proportional to $I_{\max }-A_{a}^{2} /|\Delta|^{2}$ so that the maxima and minima in the determinant $\Delta$ control the respective minima and maxima in the transmission spectrum. As shown in Fig. $\mathbf{W}$ (b) this expression fits the experimental data very well. A further analysis of the expression for the determinant yields an approximate expression for the resonant frequencies that enables identification of the key factors that control each feature in the scattering spectrum (Davis et al., 2012).

\section{CONCLUDING REMARKS}

As we have shown, the electrostatic eigenmode theory is capable of providing useful physical insights into the optical properties of ensembles of metal particles exhibiting localised surface plasmon resonances. It can, furthermore, be used to describe the interaction of metal nanoparticles with single molecules (Davis et al., 2010a, b; Gómez et all, 2012) and chiral media (Davis and Gómez, 2014). However, the theory is not without obvious limitations. The method does not include the effects of wave propagation through the background dielectric from one portion of the metal surface to another. It is assumed that all interactions occur simultaneously. In reality, the propagation delays introduce phase shifts, an effect usually termed retardation that has the effect of shifting the actual resonances to lower frequencies, or the red end of the spectrum, compared to the electrostatic result ([i]

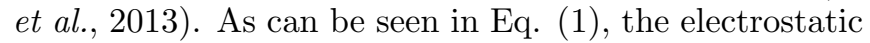
result requires that $k r \ll 1$.

The electrostatic result can be derived from an order expansion of Maxwell's equations (Mayergoyz et al., [2005) that identifies the relative importance of the different electromagnetic fields in the electrostatic problem. In essence, the zero-th order term is the interaction between the electric fields and the dielectrics, which results in the electrostatic formulation. The first-order term gives the magnetic field created by the oscillating charge distributions. This, in itself, does not affect the electrostatic result and means that we can use the electrostatic equations to predict the magnetic fields associated with localised surface plasmons, such as enhanced chirality of electromagnetic fields (Davis and Hendry, 2013). The second-order term represents the electric induction created by the time-varying first-order magnetic field, which interacts back on the surface charges. It is the neglect of this and higher terms that limits the electrostatic method. These higher-order terms are small when the dimension $d$ of the plasmonic system obeys $\epsilon_{b}(k d)^{2} \ll 1$. While this expression provides an estimate of the region of applicability, it has been shown (Davis et al. , 20019c) by comparing electrostatic predictions with numerical simulations of metal rods that the electrostatic formulation predicts resonances with errors less than $20 \%$ despite $\epsilon_{b}(k d)^{2}=2.6$ and the errors in locating resonances are generally below $5 \%$ when $\epsilon_{b}(k d)^{2}<1$.

Since the electrostatic method does not include the effects of interactions of magnetic fields, the method is unable to predict the properties of structures that exploit magnetism, such as split-ring resonators (Lezec et al., 2007). Nevertheless, the method appears to encompass most of the important physics associated with interacting metal particles and is capable of predicting the important features and behaviours of LSP spectra. Moreover, if experimental values are used for the line widths and resonance frequencies, the electrostatic method gives quite accurate predictions of the effects of LSP coupling on the geometry of the particle ensemble.

\section{References}

Abasahl, B., C. Santschi, and O. Martin, 2014, ACS Photonics 1(5), 403 .

Ashcroft, N., and N. Mermin, 1976, Solid state physics (Saunders College).

Barnes, W. L., A. Dereux, and T. W. Ebbesen, 2003, Nature 424(6950), 824.

Bergman, D., 1979a, J. Phys. C: Solid State Phys. 12, 4947.

Bergman, D., 1979b, Phys. Rev. B 19(4), 2359.

Bergman, D. J., 1978, Physics Reports 43, 377.

Bergman, D. J., 1979c, J. Phys C 12, 4947.

Bergman, D. J., 1982, J. Phys C 15, 2033.

Bergman, D. J., 1992, Solid State Physics 45, 147.

Bohren, C., and D. Huffman, 1983, Absorption and scattering of light by small particles (John Wiley and Sons, New York).

Böttcher, C., O. van Belle, P. Bordewijk, and A. Rip, 1978, Theory of electric polarization (Elsevier Scientific Pub. Co.).

Bozhevolnyi, S. I., V. S. Volkov, E. Devaux, J.-Y. Laluet, and T. W. Ebbesen, 2006, Nature 440(7083), 508.

Brandl, D. W., N. A. Mirin, and P. Nordlander, 2006, The Journal of Physical Chemistry B 110(25), 12302. 
Chang, D. E., A. S. Sorensen, P. R. Hemmer, and M. D. Lukin, 2006, Phys Rev Lett 97(5), 053002 (pages 4).

Cohen-Tannoudji, C., B. Diu, and F. Laloe, 1977, Quantum Mechanics, volume 1 (John Wiley and Sons, New York).

Davis, T., K. Vernon, and D. Gómez, 2009a, J. Appl. Phys. 106(4), 043502.

Davis, T. J., and D. E. Gómez, 2014, Phys. Rev. B 90, 235424.

Davis, T. J., D. E. Gómez, and F. Eftekhari, 2014, Optics Letters 39(16), 4938.

Davis, T. J., D. E. Gómez, and K. C. Vernon, 2010a, Phys. Rev. B 82, 205434.

Davis, T. J., D. E. Gómez, and K. C. Vernon, 2010b, Phys. Rev. B 81(4), 045432.

Davis, T. J., D. E. Gómez, and K. C. Vernon, 2010c, Nano Lett. 10(7), 2618.

Davis, T. J., and E. Hendry, 2013, Physical Review B 87(8), 085405.

Davis, T. J., M. Hentschel, N. Liu, and H. Giessen, 2012, ACS Nano 6(2), 1291.

Davis, T. J., K. C. Vernon, and D. E. Gómez, 2009b, Phys. Rev. B 79(15), 155423.

Davis, T. J., K. C. Vernon, and D. E. Gómez, 2009c, Optics Express 17(26), 23655.

Dregely, D., R. Taubert, J. Dorfmuller, R. Vogelgesang, K. Kern, and H. Giessen, 2011, Nat Commun 2, 267.

Ebbesen, T. W., C. Genet, and S. I. Bozhevolnyi, 2008, Phys. Today $\mathbf{6 1}, 44$.

Eftekhari, F., D. Gómez, and T. Davis, 2014, Optics Letters 39(10), 2994.

Engheta, N., 2007, Science 317(5845), 1698.

Engheta, N., A. Salandrino, and A. Alù, 2005, Physical Review Letters 95, 95504.

Fakonas, J. S., H. Lee, Y. A. Kelaita, and H. A. Atwater, 2014, Nat Photon 8(4), 317.

Fano, U., 1961, Phys. Rev. 124(6), 1866.

Funston, A., C. Novo, T. Davis, and P. Mulvaney, 2009, Nano Lett. 9(4), 1651.

Gallinet, B., T. Siegfried, H. Sigg, P. Nordlander, and O. J. F. Martin, 2013, Nano Letters 13(2), 497.

Gómez, D. E., A. Roberts, T. J. Davis, and K. C. Vernon, 2012, Phys. Rev. B 86, 035411.

Gómez, D. E., Z.-Q. Teo, M. Altissimo, T. J. Davis, S. Earl, and A. Roberts, 2013, Nano Letters 13, 3722.

Gómez, D. E., K. C. Vernon, and T. J. Davis, 2010, Phys. Rev. B 81(7), 075414.

Halas, N. J., S. Lal, W.-S. Chang, S. Link, and P. Nordlander, 2011, Chemical Reviews 111(6), 3913.

Haynes, C., and R. P. Van Duyne, 2001, J. Phys. Chem. B 105(24), 5599.

Hohenester, U., and A. Trugler, 2012, Computer Physics Communications 183(2), 370 .

Hokari, R., Y. Kanamori, and K. Hane, 2014, J. Opt. Soc. Am. B 31(5), 1000 .

Jackson, J. D., 1975, Classical electrodynamics (John Wiley \& Sons Inc, New York), 2 edition.

Jain, P., S. Eustis, and M. El-Sayed, 2006, J. Phys. Chem. B 110(37), 18243.

Kantor, Y., and D. Bergman, 1982, J. Phys. C: Solid State Phys. 15, 2033.

Kellog, O. D., 1929, Foundations of Potential Theory (Frederick Ungar Publishing Co, New York).

Kim, T. J., T. Thio, T. W. Ebbesen, D. E. Grupp, and H. J. Lezec, 1999, Opt Lett 24(4), 256.

Kittel, C., 1956, Introduction to Solid State Physics (Wiley,
New York), 2nd edition.

Kosako, T., Y. Kadoya, and H. F. Hofmann, 2010, Nature Photonics 4(0), 312 .

Kreibig, U., and M. Vollmer, 1995, Optical properties of metal clusters, Springer series in materials science 25 (Springer Verlag, Berlin).

Lalanne, P., and M. P. Jurek, 1998, Journal of Modern Optics 45(7), 1357.

Lalanne, P., and G. M. Morris, 1996, J. Opt. Soc. Am. A 13(4), 779 .

Langguth, L., A. H. Schokker, K. Guo, and A. F. Koenderink, 2015, Phys. Rev. B 92, 205401.

Lezec, H. J., J. A. Dionne, and H. A. Atwater, 2007, Science 316(5823), 430.

Li, Y., K. Zhao, H. Sobhani, K. Bao, and P. Nordlander, 2013, The Journal of Physical Chemistry Letters 4(8), 1352.

Li, Z., S. Butun, and K. Aydin, 2014, ACS Nano 8(8), 8242.

Liu, N., M. Hentschel, T. Weiss, A. P. Alivisatos, and H. Giessen, 2011, Science 332(6036), 1407.

Liu, N., L. Langguth, T. Weiss, J. K astel, M. Fleischhauer, T. Pfau, and H. Giessen, 2009, Nature Materials 8, 758.

Liu, N., F. Wen, Y. Zhao, Y. Wang, P. Nordlander, N. Halas, and A. Alù, 2013, Nano Letters 13(1), 142 .

Liu, Z., J. M. Steele, W. Srituravanich, Y. Pikus, C. Sun, and X. Zhang, 2005, Nano Letters 5(9), 1726.

Lovera, A., B. Gallinet, P. Nordlander, and O. J. Martin, 2013, ACS Nano 7(5), 4527.

Luk'yanchuk, B., N. I. Zheludev, S. A. Maier, N. J. Halas, P. Nordlander, H. Giessen, and C. T. Chong, 2010, Nat Mater 9(9), 707.

Maier, S., 2007, Plasmonics: fundamentals and applications (Springer, New York).

Mayergoyz, I. D., D. R. Fredkin, and Z. Zhang, 2005, Phys. Rev. B 72(15), 155412.

Mayergoyz, I. D., Z. Zhang, and G. Miano, 2007, Phys. Rev. Lett. 98(14), 147401.

Meystre, P., and M. Sargent, 1998, Elements of Quantum Optics (Springer Berlin Heidelberg).

Mie, G., 1908, Annalen der Physik 330(3), 377.

Ming, T., L. Zhao, Z. Yang, H. Chen, L. Sun, J. Wang, and C. Yan, 2009, Nano Letters 9(11), 3896 .

Mirin, N. A., K. Bao, and P. Nordlander, 2009, J Phys Chem A 113(16), 4028.

Miroshnichenko, A. E., S. Flach, and Y. S. Kivshar, 2010, Rev. Mod. Phys. 82, 2257.

Moharam, M. G., T. K. Gaylord, E. B. Grann, and D. A. Pommet, 1995, J. Opt. Soc. Am. A 12(5), 1068.

Mojarad, N. M., and M. Agio, 2009, Opt. Express 17(1), 117.

Nordlander, P., C. Oubre, E. Prodan, K. Li, and M. Stockman, 2004, Nano Lett. 4(5), 899.

Odom, T. W., and G. C. Schatz, 2011, Chemical Reviews $\mathbf{1 1 1}(6), 3667$.

Ouyang, F., and M. Isaacson, 1989, Phil. Mag. B 60(4), 481.

Ozbay, E., 2006, Science 311(5758), 189.

Pacifici, D., H. Lezec, and H. Atwater, 2007, Nature Photonics 1, 402.

Prodan, E., and P. Nordlander, 2004, J. Chem. Phys. 120, 5444 .

Prodan, E., C. Radloff, N. Halas, and P. Nordlander, 2003, Science 302, 419 .

Raether, H., 1977, in Physics of Thin Films, edited by G. Hass, M. H. Francombe, and R. W. Hoffman (Academic Press), volume 9, pp. $145-261$.

Raether, H., 1988, Surface Plasmons on Smooth and Rough 
Surfaces and on Gratings, Springer Tracts in Modern Physics (Springer Berlin Heidelberg).

Rechberger, W., A. Hohenau, A. Leitner, J. Krenn, B. Lamprecht, and F. Aussenegg, 2003, Opt. Commun. 220, 137

Sancho-Parramon, J., and S. Bosch, 2012, ACS Nano 6(9), 8415.

Schouten, H. F., N. Kuzmin, G. Dubois, T. D. Visser, G. Gbur, P. F. A. Alkemade, H. Blok, G. W. t. Hooft, D. Lenstra, and E. R. Eliel, 2005, Phys. Rev. Lett. 94, 053901.

Sönnichsen, C., T. Franzl, T. Wilk, G. von Plesson, J. Feldmann, O. Wilson, and P. Mulvaney, 2002, Physical Review Letters 88(7), 077402.

Su, K., Q. Wei, X. Zhang, J. Mock, D. Smith, and S. Schultz, 2003, Nano Lett. 3(8), 1087.

Sun, Y., B. Edwards, A. Alu, and N. Engheta, 2012, Nature Materials 11(3), 208 .

Taubert, R., M. Hentschel, J. Kästel, and H. Giessen, 2012, Nano Letters 12, 13671371.

Thaxton, C., and C. Mirkin, 2005, Nat. Biol. 23, 681 .

Weaver, J. H., and H. P. R. Frederikse, 2006, in CRC Handbook of Chemistry and Physics (CRC Press), 87 edition.

Wei, H., A. Reyes-Coronado, P. Nordlander, J. Aizpurua, and H. Xu, 2010, ACS Nano 4(5), 2649, pMID: 20397629.

Weiss, T., N. A. Gippius, S. G. Tikhodeev, G. Granet, and H. Giessen, 2009, Journal of Optics A: Pure and Applied Optics 11(11), 114019.

Wokaun, A., J. P. Gordon, and P. F. Liao, 1982, Phys. Rev. Lett. 48(14), 957.

Yang, S.-C., H. Kobori, C.-L. He, M.-H. Lin, H.-Y. Chen, C. Li, M. Kanehara, T. Teranishi, and S. Gwo, 2010, Nano Letters 10(2), 632 .

Zayats, A., I. Smolyaninov, and A. Maradudin, 2005, Phys. Rep. 408, 131 .

Zhang, S., D. Genov, Y. Wang, M. Liu, and X. Zhang, 2008, Physical Review Letters 101, 47401. 


\section{Appendix S1: The eigenmodes and eigenvalues of Eqn. (四) for a sphere}

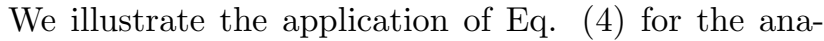
lytical description of a metallic nano-sphere in terms of eigenmodes and its interaction with an external electric field. Some of the results found in this section have also been applied for describing the interaction of molecules with metal nanoparticles (Davis et al, 2010) $)$.

The starting point is to expand the surface charge density using the spherical harmonics as a basis set(Wackson, [1.975):

$$
\sigma(\mathbf{r})=\sum_{k, n} \sigma_{k, n}(R) Y_{k, n}(\Omega)
$$

where $\Omega=\theta, \phi$ denotes the solid angle in the sphere which is considered to have a radius $R . \sigma_{k, n}(R)=$ $A_{k, n} R^{k}+B_{k, n} R^{-(k+1)}$ is the radial part with coefficients as yet undetermined and the second index in the summation $n=-k,-|k-1|, \cdots, 0, \cdots, k-1, k$.

Equation (四) can be also expressed as:

$$
\sigma(\mathbf{r})=-\frac{\gamma}{2 \pi} \oint \sigma\left(\mathbf{r}^{\prime}\right) \hat{n} \cdot \nabla \frac{1}{\left|\mathbf{r}-\mathbf{r}^{\prime}\right|} d S^{\prime},
$$

and the term $1 /\left|\mathbf{r}-\mathbf{r}^{\prime}\right|$ can be also expanded using spherical harmonics using the well-known expression (Wackson, [1.975):

$$
\frac{1}{\left|\mathbf{r}-\mathbf{r}^{\prime}\right|}=4 \pi \sum_{l, m} \frac{1}{2 l+1} \frac{r<^{l}}{r>^{l+1}} Y_{l, m}^{*}\left(\Omega^{\prime}\right) Y_{l, m}(\Omega) .
$$

Substituting this and Eq. (5]) into Eq. (52):

$$
\begin{aligned}
\sigma(\mathbf{r}) & =-\frac{\gamma}{2 \pi} \sum_{k, n} \sum_{l, m} \frac{4 \pi}{2 l+1} \\
& \int_{0}^{2 \pi} d \phi^{\prime} \int_{0}^{\pi} d \theta^{\prime} \sin \left(\theta^{\prime}\right) Y_{l, m}^{*}\left(\Omega^{\prime}\right) Y_{l, m}(\Omega) Y_{k, n}\left(\Omega^{\prime}\right) \\
& \times R^{2} \sigma_{k, n}(R) \hat{n} \cdot \nabla \frac{r<^{l}}{r>^{l+1}} \\
& =\frac{\gamma}{2 \pi} \sum_{k, n} \sum_{l, m} \frac{4 \pi}{2 l+1} \delta_{k, l} \delta_{n, m} R^{2} \sigma_{k, n}(R) \frac{1}{2 R^{2}} \\
& =\frac{\gamma}{2 k+1} \sum_{k, n} \sigma_{k, n}(R) Y_{k, n}(\Omega),
\end{aligned}
$$

which according to Eq. (ST]) imply that the eigenvalues $\gamma$ are thus:

$$
\gamma=2 k+1
$$

A requirement of charge neutrality for the surface charge distribution reads:

$$
\begin{aligned}
\oint \sigma(\mathbf{r}) d S & =0 \\
& =\sum_{l, m} \int_{0}^{2 \pi} d \phi \int_{0}^{\pi} d \theta \sin (\theta) R^{2} \sigma_{l, m}(R) Y_{l, m}(\Omega) \\
& =\sum_{l, m} \int_{0}^{2 \pi} d \phi \int_{0}^{\pi} d \theta \sin (\theta) Y_{l, m}(\Omega) Y_{0,0}(\Omega) \\
& \times R^{2} \sigma_{l, m}(R) \sqrt{4 \pi} \\
& =\sum_{l, m} \delta_{l, 0} \delta_{m, 0} R^{2} \sigma_{l, m}(R) \sqrt{4 \pi}
\end{aligned}
$$

a condition only satisfied if $\sigma_{0,0}(R)=0$, i.e. the expansion of Eq. (ST) is only made for $k>0$. Therefore, for a spherical object, the eigenmodes of Eq. (四) are $\sigma_{k, n}(R) Y_{k, n}(\Omega)$ with eigenvalues $2 k+1$ (each being $2 k+1$ degenerate) where the index $k=1,2, \cdots$.

The solutions to Eq. (目) can also found by following the same procedure. However, as stated in section $\mathbb{~}$, the solutions to Eqs. (田) and (四) form a bi-orthogonal set, for which $\oint \tau_{m}^{i}(\mathbf{r}) \sigma_{n}^{j}(\mathbf{r}) d S=\delta_{m n} \delta^{i j}$, requiring that the functional form of $\tau_{m}^{i}(\mathbf{r})$ is $\tau_{m}^{i}(\mathbf{r})=\tau_{m}^{i}(R) Y_{m l}^{*}(\Omega)$ (with $l=-m,-|m-1|, \cdots, 0, \cdots, m-1, m)$ :

$$
\begin{aligned}
\delta_{m n} \delta^{i j} & =\oint \tau_{m}^{i}(\mathbf{r}) \sigma_{n}^{j}(\mathbf{r}) d S \\
& =\int_{0}^{2 \pi} d \phi \int_{0}^{\pi} d \theta \sin (\theta) R^{2} \tau_{m}^{i}(R) \sigma_{n}^{j}(R) Y_{m l}^{*}(\Omega) Y_{n k}(\Omega) \\
& =R^{2} \tau_{m}^{i}(R) \sigma_{n}^{j}(R) \delta_{m n} \delta_{l k},
\end{aligned}
$$

which imply thus $\delta^{i j}=R^{2} \tau_{m}^{i}(R) \sigma_{m}^{j}(R)$.

We now proceed to analyse the interaction of a metal sphere with an uniform incident electric field, polarised along the $\hat{z}$ axis. Due to this interaction, the sphere will support an LSP described by a superposition of eigenmodes $\sigma(\vec{r})=\sum_{m} a_{m}(\omega) \sigma_{m}(\mathbf{r})$, where the excitation amplitude $a_{m}(\omega)$ of the $m$-th eigenmode is given by Eq. (प) of section ㅁ::

$$
\begin{aligned}
a_{m}(\omega) & =f_{m}(\omega) \oint \tau_{m}(\mathbf{r}) \hat{n} \cdot \mathbf{E}_{0}(\mathbf{r}) d S \\
& =f_{m}(\omega) \oint \tau_{m}(\mathbf{r}) E_{0} \cos (\theta) d S \\
& =\sum_{l=-m}^{m} f_{m}(\omega) E_{0} \sqrt{\frac{4 \pi}{3}} \int_{0}^{2 \pi} d \phi \int_{0}^{\pi} d \theta \sin (\theta) \\
& \times R^{2} \tau_{m}(R) Y_{m l}^{*}(\Omega) Y_{1,0}(\Omega) \\
& =f_{m}(\omega) E_{0} \sqrt{\frac{4 \pi}{3}} R^{2} \tau_{m}(R) \delta_{m 1} \delta_{l 0}
\end{aligned}
$$

meaning that under this illumination, the surface charge 
distribution describing the sphere's LSP is:

$$
\begin{aligned}
\sigma(\mathbf{r}) & =\sum_{m} f_{m}(\omega) E_{0} \sqrt{\frac{4 \pi}{3}} R^{2} \tau_{m}(R) \sigma_{m}(R) Y_{m l}(\Omega) \delta_{m 1} \delta_{l 0} \\
& =f_{m=1}(\omega) E_{0} \sqrt{\frac{4 \pi}{3}} Y_{10}(\Omega) \\
& =\frac{3 \epsilon_{b}\left(\epsilon_{m}(\omega)-\epsilon_{b}\right)}{2 \epsilon_{b}+\epsilon_{m}(\omega)} E_{0} \sqrt{\frac{4 \pi}{3}} Y_{10}(\Omega)
\end{aligned}
$$

which is the result found in by directly solving Laplace equation in spherical coordinates (Böttcher et al, ए978).

The dipole moment $\mathbf{p}$ associated with this charge distribution is found by performing the surface integral $\oint \sigma(\mathbf{r}) \mathbf{r} d S$, recognising that $\mathbf{r}=r(\sin (\theta) \cos (\phi) \hat{x}+\sin (\theta) \sin (\phi) \hat{y}+\cos (\theta) \hat{z}):$

$$
\begin{aligned}
\mathbf{p} & =a_{m=1}(\omega) \oint \sigma_{m=1}(R) Y_{10}(\Omega) \mathbf{r} d S \\
& =a_{m=1}(\omega) \int_{0}^{2 \pi} d \phi \int_{0}^{\pi} d \theta \sin (\theta) Y_{10}(\Omega) Y_{10}(\Omega) \\
& \times R^{3} \sigma_{m=1}(R) \sqrt{\frac{4 \pi}{3}} \hat{z} \\
& =a_{m=1}(\omega) R^{3} \sigma_{m=1}(R) \sqrt{\frac{4 \pi}{3}} \hat{z}
\end{aligned}
$$

and substitution of the expression for the excitation amplitude, bearing in mind the bi-orthogonality condition:

$$
\begin{aligned}
\mathbf{p} & =\frac{4 \pi R^{3}}{3} f_{m=1}(\omega) E_{0}\left[R^{2} \tau_{1}(R) \sigma_{1}(R)\right] \hat{z} \\
& =\frac{3 \epsilon_{b}\left(\epsilon_{m}(\omega)-\epsilon_{b}\right)}{2 \epsilon_{b}+\epsilon_{m}(\omega)} \frac{4 \pi R^{3}}{3} E_{0} \hat{z},
\end{aligned}
$$

which is also in agreement with well-known results (Böttcher et al., 1978) but more importantly, it permits for a physical interpretation of the factor $f_{m}(\omega)$ : the polarisability per unit volume of the $m$-th eigenmode.

\section{Appendix S2: Drude model and the electostatic approximation}

According to the Drude model, the permittivity of a metal can be approximated by:

$$
\epsilon(\omega)=\epsilon_{\infty}^{D}-\frac{\omega_{P}^{2}}{\omega\left(\omega+i \Gamma_{m}\right)} .
$$

In order to get a closed analytical form of the factor $f$ of Eq. ( $(\mathbb{Q})$, we consider the case when the frequency is close to a localised surface plasmon resonance $\omega=\omega_{m}+\delta$ and furthermore assume that $\left(\delta / \omega_{m}\right)^{2} \ll 1$ :

$$
\begin{aligned}
\epsilon(\omega) & =\epsilon_{\infty}^{D}-\frac{\omega_{P}^{2}}{\left(\omega_{m}+\delta\right)\left(\omega_{m}+\delta+i \Gamma_{m}\right)} \\
& \approx \epsilon_{\infty}^{D}-\frac{\omega_{P}^{2}}{\omega_{m}^{2}\left(1+\frac{2 \delta}{\omega_{m}}+\frac{i \Gamma_{m}}{\omega_{m}}\right)} .
\end{aligned}
$$

A Taylor expansion of the term in brackets in the denominator results in

$$
\begin{aligned}
\epsilon(\omega) & \approx \epsilon_{\infty}^{D}-\frac{\omega_{P}^{2}}{\omega_{m}^{2}\left(1+\frac{2 \delta}{\omega_{m}}+\frac{i \Gamma_{m}}{\omega_{m}}\right)} \\
& \approx \epsilon_{\infty}^{D}-\frac{\omega_{P}^{2}}{\omega_{m}^{2}}\left(1-\frac{2 \delta}{\omega_{m}}-\frac{i \Gamma_{m}}{\omega_{m}}\right) \\
& \approx \epsilon_{\infty}^{D}-\frac{\omega_{P}^{2}}{\omega_{m}^{2}}+\frac{2 \omega_{P}^{2}}{\omega_{m}^{3}}\left(\omega-\omega_{m}+i \Gamma_{m} / 2\right),
\end{aligned}
$$

where we have kept only the first order terms

With this result we now consider $f$

$$
\begin{aligned}
f_{m}^{j}(\omega) & \approx-\frac{2 \epsilon_{b}^{2}\left(\gamma_{m}^{j}\right)^{2}}{\left(\gamma_{m}^{j}-1\right)^{2}} \frac{\left(\omega_{m}^{j}\right)^{3}}{\omega_{P}^{2}} \frac{1}{\omega-\omega_{m}^{j}+i \Gamma_{m}^{j} / 2} \\
& =-\frac{A_{m}^{j}}{\omega-\omega_{m}^{j}+i \Gamma_{m}^{j} / 2}
\end{aligned}
$$

where Eq. (52) has been used. 